\title{
Black Hole on a Chip: Proposal for a Physical Realization of the Sachdev-Ye-Kitaev model in a Solid-State System
}

\author{
D. I. Pikulin ${ }^{1}$ and M. Franz ${ }^{2}$ \\ ${ }^{1}$ Station Q, Microsoft Research, Santa Barbara, California 93106-6105, USA \\ ${ }^{2}$ Department of Physics and Astronomy, University of British Columbia, \\ Vancouver, British Columbia, Canada V6T 1Z1 \\ and Quantum Matter Institute, University of British Columbia, \\ Vancouver British Columbia, Canada V6T 1Z4
}

(Received 14 February 2017; revised manuscript received 24 May 2017; published 13 July 2017)

\begin{abstract}
A system of Majorana zero modes with random infinite-range interactions-the Sachdev-Ye-Kitaev (SYK) model - is thought to exhibit an intriguing relation to the horizons of extremal black holes in two-dimensional anti-de Sitter space. This connection provides a rare example of holographic duality between a solvable quantum-mechanical model and dilaton gravity. Here, we propose a physical realization of the SYK model in a solid-state system. The proposed setup employs the Fu-Kane superconductor realized at the interface between a three-dimensional topological insulator and an ordinary superconductor. The requisite $N$ Majorana zero modes are bound to a nanoscale hole fabricated in the superconductor that is threaded by $N$ quanta of magnetic flux. We show that when the system is tuned to the surface neutrality point (i.e., chemical potential coincident with the Dirac point of the topological insulator surface state) and the hole has sufficiently irregular shape, the Majorana zero modes are described by the SYK Hamiltonian. We perform extensive numerical simulations to demonstrate that the system indeed exhibits physical properties expected of the SYK model, including thermodynamic quantities and two-point as well as four-point correlators, and discuss ways in which these can be observed experimentally.
\end{abstract}

DOI: 10.1103/PhysRevX.7.031006

Subject Areas: Condensed Matter Physics,
Interdisciplinary Physics,
Superconductivity,
Topological Insulators

\section{INTRODUCTION}

Models of particles with infinite-range interactions have a long history in nuclear physics dating back to the pioneering works of Wigner [1] and Dyson [2] and in condensed matter physics in studies describing spin glass and spin liquid states of matter [3-5]. More recently, Kitaev [6] and Maldacena and Stanford [7] formulated and studied a Majorana fermion version of the model with all-to-all random interactions first proposed by Sachdev and Ye [4]. The resulting Sachdev-Ye-Kitaev (SYK) model, defined by the Hamiltonian Eq. (1.1), is solvable in the limit of large number $N$ of fermions and exhibits a host of intriguing properties. The SYK model is believed to be holographic dual of extremal black hole horizons in two-dimensional anti-de Sitter $\left(\mathrm{AdS}_{2}\right)$ space and has been argued to possess remarkable connections to information theory, many-body thermalization, and quantum chaos [8-13]. Various extensions of the SYK model have been put forth containing

Published by the American Physical Society under the terms of the Creative Commons Attribution 4.0 International license. Further distribution of this work must maintain attribution to the author(s) and the published article's title, journal citation, and DOI. supersymmetry [14], interesting quantum phase transitions $[15,16]$, and higher-dimensional extensions [17,18], as well as a version that does not require randomness [19]. Given its fascinating properties it would be of obvious interest to have an experimental realization of the SYK model or its variants. Thus far a realization of the Sachdev-Ye model (with complex fermions) has been proposed using ultracold gases [20], and a protocol for digital quantum simulation of both the complex and Majorana fermion versions of the model has been discussed [21]. A natural realization of the SYK model in a solid-state system is thus far lacking.

Recent years have witnessed numerous proposals for experimental realizations of unpaired Majorana zero modes in solid-state systems [22-26], with compelling experimental evidence for their existence gradually mounting in several distinct platforms [27-35]. The purpose of this paper is to propose a physical realization of the SYK model in one of these platforms. The SYK Hamiltonian we implement is given by

$$
\mathcal{H}_{\mathrm{SYK}}=\sum_{i<j<k<l} J_{i j k l} \chi_{i} \chi_{j} \chi_{k} \chi_{l}
$$

where $J_{i j k l}$ are random independent coupling constants and $\chi_{j}$ represent the Majorana zero-mode operators that obey the canonical anticommutation relations 


$$
\left\{\chi_{i}, \chi_{j}\right\}=\delta_{i j}, \quad \chi_{j}^{\dagger}=\chi_{j}
$$

The proposed device, depicted in Fig. 1, employs an interface between a 3D topological insulator (TI) and an ordinary superconductor such as $\mathrm{Nb}$ or $\mathrm{Pb}$. Fu and Kane [36] showed theoretically that magnetic vortices in such an interface host unpaired Majorana zero modes, and signatures consistent with this prediction have been reported in $\mathrm{Bi}_{2} \mathrm{Te}_{3} / \mathrm{NbSe}_{2}$ heterostructures [34,35]. Under ordinary circumstances these vortices tend to form an Abrikosov lattice and the low-energy effective theory is dominated by two-fermion terms $i K_{i j} \chi_{i} \chi_{j}$, with the hopping amplitudes $K_{i j}$ decaying exponentially with the distance between vortex sites $\left|\boldsymbol{r}_{i}-\boldsymbol{r}_{j}\right|$. Four-fermion interaction terms of the type required to implement the SYK Hamiltonian Eq. (1.1) are generically also present but are subdominant and also decay exponentially with distance. Realizing the SYK model in this setup therefore entails two key challenges: (i) one must find a way to suppress the two-fermion tunneling terms and (ii) render the four-fermion interactions effectively infinite ranged. In addition, the four-fermion coupling constants $J_{i j k l}$ must be sufficiently random. In the following we show how these challenges can be overcome by judicious engineering of various aspects of the device depicted in Fig. 1.

The first challenge can be met by tuning the surface state of the TI into its global neutrality point such that the chemical potential $\mu$ lies at the Dirac point. At the neutrality point the interface superconductor is known to acquire an extra chiral symmetry which prohibits any two-fermion terms [37]. In other words, the symmetry requires $K_{i j}=0$ and the low-energy Hamiltonian is then dominated by the four-fermion terms [38]. The second requirement of effectively infinite-ranged interactions can be satisfied by localizing all Majorana zero modes in the same region of space. In our proposed device this is achieved by fabricating a hole in the superconducting (SC) layer, as illustrated in Fig. 1. If the sample is cooled in a weak applied magnetic field, an integer number $N$ of magnetic

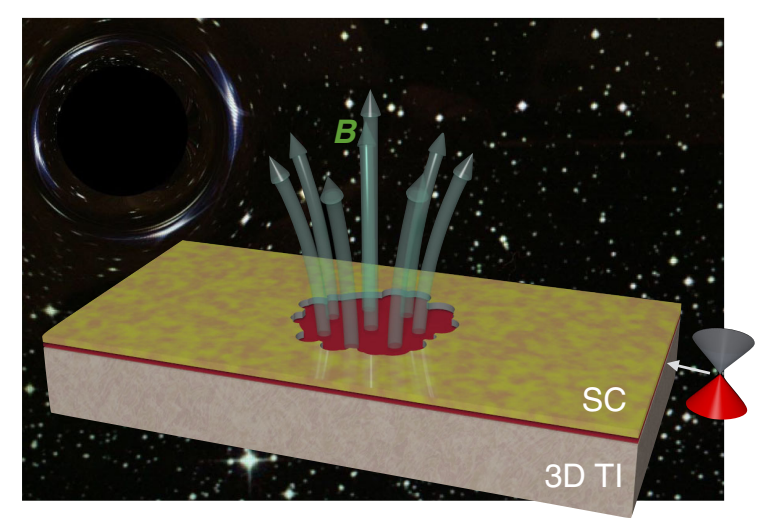

FIG. 1. The proposed setup for a solid-state realization of the SYK model. flux quanta can be trapped in the hole. The SC phase $\theta$ will then wind by $2 \pi N$ around the hole, forming effectively an $N$-fold vortex with $N$ Majorana zero modes bound to the hole. If, furthermore, the hole is designed to have an irregular shape, the Majorana wave functions will have random spatial structure and their overlaps will give rise to the required randomness in the coupling constants $J_{i j k l}$. This randomness is related to random classical trajectories inside such a hole, or "billiards," as it is commonly called in the quantum chaos literature $[39,40]$. We note a related proposal to realize the SYK model using semiconductor quantum wires coupled to a disordered quantum dot advanced in the recent work [41].

In the rest of the paper we provide the necessary background on our proposed system and support its relation to the SYK model by physical arguments and by detailed model calculations. We first review the Fu-Kane model [36] for the TI-SC interface and numerically calculate the Majorana wave functions localized in a hole threaded by $N$ magnetic flux quanta in the presence of disorder. Assuming that the constituent electrons interact via screened Coulomb potential, we then explicitly calculate the four-fermion coupling constants $J_{i j k l}$ between the Majorana zero modes. We, finally, use these as input data for the many-body Majorana Hamiltonian which we diagonalize numerically for $N$ up to 32 and study its thermodynamic properties, level statistics, as well as twoand four-point correlators. We show that these behave precisely as expected of the SYK model with random independent couplings. We also discuss the effect of small residual two-fermion terms that will inevitably be present in a realistic device and propose ways to experimentally detect signatures of the SYK physics using tunneling spectroscopy.

\section{SYK MODEL FROM INTERACTING MAJORANA ZERO MODES AT THE TI-SC INTERFACE}

\section{A. Fu-Kane superconductor}

The surface of a canonical 3D TI, such as $\mathrm{Bi}_{2} \mathrm{Se}_{3}$, hosts a single massless Dirac fermion protected by time-reversal symmetry. When placed in the proximity of an ordinary superconductor, the surface state is described by the Fu-Kane Hamiltonian [36],

$$
\mathcal{H}_{\mathrm{FK}}=\int d^{2} r \hat{\Psi}_{r}^{\dagger} H_{\mathrm{FK}}(\boldsymbol{r}) \hat{\Psi}_{r}
$$

where $\hat{\Psi}_{r}=\left(c_{\uparrow r}, c_{\downarrow r}, c_{\downarrow r}^{\dagger},-c_{\uparrow_{r}}^{\dagger}\right)^{T}$ is the Nambu spinor and

$H_{\mathrm{FK}}=\tau^{z}\left[v_{F} \boldsymbol{\sigma} \cdot\left(\boldsymbol{p}-\tau^{z} \frac{e}{c} \boldsymbol{A}\right)-\mu\right]+\tau^{x} \Delta_{1}-\tau^{y} \Delta_{2}$.

Here, $v_{F}$ is the velocity of the surface state, $\boldsymbol{p}=-i \hbar \boldsymbol{\nabla}$ denotes the momentum operator, $\Delta=\Delta_{1}+i \Delta_{2}$ is the SC 
order parameter, and $\boldsymbol{\sigma}, \boldsymbol{\tau}$ are Pauli matrices in spin and Nambu spaces, respectively. To describe the geometry depicted in Fig. 1, we take

$$
\Delta(\boldsymbol{r})= \begin{cases}0 & r<R(\varphi) \\ \Delta_{0} e^{i N \varphi} & r>R(\varphi),\end{cases}
$$

where $\varphi$ is the polar angle and $R(\varphi)$ denotes the hole radius. The vector potential is taken to yield total flux through the hole $\oint_{C} d \mathbf{l} \cdot \boldsymbol{A}=N \Phi_{0}$, with $\Phi_{0}=h c / 2 e$ the SC flux quantum and the contour $C$ taken to encircle the hole at a radius well beyond the effective magnetic penetration depth of the SC film, $\lambda_{\text {eff }}=2 \lambda_{L}^{2} / d$. (Here, $\lambda_{L}$ is the London penetration depth of the bulk SC and $d$ the film thickness.)

The Hamiltonian Eq. (2.2) respects the particle-hole (p-h) symmetry generated by $\Xi=\sigma^{y} \tau^{y} \mathcal{K}\left(\Xi^{2}=+1\right)$, where $\mathcal{K}$ denotes complex conjugation. For a purely real gap function $\Delta$ and zero magnetic field $\boldsymbol{B}=\boldsymbol{\nabla} \times \boldsymbol{A}$, it also obeys the physical time-reversal symmetry $\Theta=i \sigma^{y} \mathcal{K}$ $\left(\Theta^{2}=-1\right)$. In the presence of vortices $\Delta$ becomes complex and the time-reversal symmetry is broken. The Fu-Kane model with vortices therefore falls into symmetry class $D$ in the Altland-Zirnbauer classification [42], which, in accordance with Ref. [37], implies a $Z_{2}$ classification for the zero modes associated with vortices. Physically, this means that a system with total vorticity $N_{V}$ will have $N=$ $\left(N_{V}\right.$ mod 2) exact zero modes, in accord with the expectation that any even number of Majorana zero modes will generically hybridize and form complex fermions at nonzero energy.

When $\mu=0$, the Hamiltonian Eq. (2.2) also respects a fictitious time-reversal symmetry generated by $\Sigma=\sigma^{x} \tau^{x} \mathcal{K}$ $\left(\Sigma^{2}=+1\right)$. It is important to note that unlike the physical time-reversal, this symmetry remains valid even in the presence of the applied magnetic field and vortices. At the neutrality point, the two symmetries $\Xi$ and $\Sigma$ define a BDI class with chiral symmetry $\Pi=\Xi \Sigma=\sigma^{z} \tau^{z}$. This, in accordance with Ref. [37], implies an integer classification of zero modes associated with point defects. A system with total vorticity $N_{V}$ will thus exhibit $N=N_{V}$ exact zero modes, irrespective of the precise geometric arrangement of the individual vortices and other details. This remains true in the presence of any disorder that does not break the $\Sigma$ symmetry. Specifically, randomness in $v_{F}$ and $\Delta$ will not split the zero modes but random contributions to $\mu$ will.

Another way to establish the existence of exact zero modes in the Hamiltonian Eq. (2.2) with $\mu=0$ is to recognize it as a version of the Jackiw-Rossi Hamiltonian [43], well known in particle physics. An index theorem for this Hamiltonian, conjectured by Jackiw and Rossi and later proven by Weinberg [44], equates the number $N$ of its exact zero modes in region $\mathcal{M}$ to the total vorticity, $N_{V}=[1 /(2 \pi)] \oint_{\partial \mathcal{M}} d \mathbf{l} \cdot \boldsymbol{\nabla} \theta$, contained in that region. A region threaded by $N_{V}$ magnetic flux quanta will thus contain $N$ exact zero modes.
In the geometry of Fig. 1 the Majorana modes we discuss above can equivalently be viewed as living at the boundary between a magnetically gapped TI surface on the inside and a SC region on the outside of the hole. The existence of such modes is well known and has been discussed in several papers $[45,46]$.

The existence and properties of the zero modes in the FuKane Hamiltonian have been extensively tested by analytic and numerical approaches for a single vortex [36], pair of vortices [47,48], periodic Abrikosov lattices [49,50], as well as the "giant vortex" geometry [51] similar to our proposed setup. This body of work firmly establishes the existence of exact Majorana zero modes for $\mu=0$ in accordance with the Jackiw-Rossi-Weinberg index theorem. Away from neutrality it is found that the zero modes are split due to two-fermion tunneling terms $K_{i j} \propto \mu$, where the constant of proportionality is related to the wave function overlap between $\chi_{i}$ and $\chi_{j}$. In addition, it has been found that for a singly quantized vortex at neutrality the zero mode is separated from the rest of the spectrum by a gap $\sim \Delta_{0}$, where $\Delta_{0}$ is the SC gap magnitude far from the vortex. We shall see that for a judiciously chosen hole size this convenient hierarchy of energy scales remains in place with $N$ zero modes separated by a large gap from the rest of the spectrum.

\section{B. Low-energy effective theory}

Having established a convenient platform that hosts $N$ Majorana zero modes with wave functions localized in the same region of space, we now proceed to derive the effective low-energy theory in terms of the Majorana zeromode operators $\chi_{j}$. To this end, we write the full secondquantized Hamiltonian of the system as

$$
\mathcal{H}=\mathcal{H}_{\mathrm{FK}}^{(N)}+\delta \mathcal{H}_{\mathrm{FK}}+\mathcal{H}_{\text {int }} .
$$

Here, $\mathcal{H}_{\mathrm{FK}}^{(N)}$ stands for the part of the Fu-Kane Hamiltonian Eq. (2.2) that obeys the fictitious time-reversal symmetry $\Sigma$ and exhibits, therefore, $N$ exact zero modes. $\delta \mathcal{H}_{\mathrm{FK}}$ contains all the remaining fermion bilinears that break $\Sigma$ such as the chemical potential term. $\mathcal{H}_{\text {int }}$ defines the four-fermion interactions that have been ignored thus far but will play a pivotal role in the physics of the SYK model we study. We assume that electrons are subject to screened Coulomb interactions described by

$$
\mathcal{H}_{\text {int }}=\frac{1}{2} \iint d^{2} r d^{2} r^{\prime} \hat{\rho}(\boldsymbol{r}) V\left(\boldsymbol{r}-\boldsymbol{r}^{\prime}\right) \hat{\rho}\left(\boldsymbol{r}^{\prime}\right),
$$

where $V(\boldsymbol{r})$ is the interaction potential and $\hat{\rho}(\boldsymbol{r})=c_{\sigma r}^{\dagger} c_{\sigma \boldsymbol{r}}$ is the electron charge density operator.

Now imagine we have solved the single-electron problem defined by the Hamiltonian $H_{\mathrm{FK}}^{(N)}$ for the device geometry sketched in Fig. 1 with $N$ flux quanta threaded 
through the hole. We thus have the complete set of singleparticle eigenfunctions $\Phi_{n}(\boldsymbol{r})$ and eigenenergies $\varepsilon_{n}$ of $H_{\mathrm{FK}}^{(N)}$. The corresponding second-quantized Hamiltonian can then be written in a diagonal form,

$$
\mathcal{H}_{\mathrm{FK}}^{(N)}=\sum_{n}^{\prime} \varepsilon_{n} \hat{\psi}_{n}^{\dagger} \hat{\psi}_{n}+E_{g},
$$

where

$$
\hat{\psi}_{n}=\int d^{2} r \Phi_{n}^{\dagger}(\boldsymbol{r}) \hat{\Psi}_{r}
$$

is the eigenmode operator belonging to the eigenvalue $\varepsilon_{n}$. The sum over $n$ is restricted to the positive energy eigenvalues and $E_{g}$ is a constant representing the ground-state energy. At the neutrality point, according to our preceding discussion, $N$ of the $\hat{\psi}_{n}$ eigenmodes coincide with the exact zero modes mandated by the Jackiw-Rossi-Weinberg index theorem. We denote these $\chi_{j}$ with $j=1, \ldots, N$. Because $\varepsilon_{j}=0$, these modes do not contribute to the Hamiltonian Eq. (2.6). The zero-mode eigenfunctions $\Phi_{j}(\boldsymbol{r})$ can be chosen as eigenstates of the p-h symmetry generator $\Xi$. They then satisfy the reality condition

$$
\sigma^{y} \tau^{y} \Phi_{j}^{*}(\boldsymbol{r})=\Phi_{j}(\boldsymbol{r}),
$$

which implies that $\chi_{j}^{\dagger}=\chi_{j}$; the zero modes are Majorana operators.

As noted before, the $N$ zero modes are separated by a gap from the rest of the spectrum. As long as $\delta \mathcal{H}_{\mathrm{FK}}$ and $\mathcal{H}_{\text {int }}$ remain small compared to this gap we may construct the effective low-energy theory of the system by simply projecting onto the part of the Hilbert space generated by $N$ Majorana zero modes. In practical terms this is accomplished by inverting Eq. (2.7) to obtain

$$
\hat{\Psi}_{r}=\sum_{n} \Phi_{n}(\boldsymbol{r}) \hat{\psi}_{n}
$$

then substituting $\hat{\Psi}_{r}$ into $\delta \mathcal{H}_{\mathrm{FK}}$ and $\mathcal{H}_{\mathrm{int}}$ and retaining only those terms that contain zero-mode operators $\chi_{j}$ but no finite-energy eigenmodes. We thus obtain

$$
\mathcal{H}_{\mathrm{eff}}=\frac{i}{2 !} \sum_{i, j} \tilde{K}_{i j} \chi_{i} \chi_{j}+\frac{1}{4 !} \sum_{i, j, k, l} \tilde{J}_{i j k l} \chi_{i} \chi_{j} \chi_{k} \chi_{l},
$$

where

$$
\begin{aligned}
i \tilde{K}_{i j} & =2 ! \int d^{2} r \Phi_{i}^{\dagger}(\boldsymbol{r}) \delta H_{\mathrm{FK}}(\boldsymbol{r}) \Phi_{j}(\boldsymbol{r}), \\
\tilde{J}_{i j k l} & =\frac{4 !}{2} \iint d^{2} r d^{2} r^{\prime} \rho_{i j}(\boldsymbol{r}) V\left(\boldsymbol{r}-\boldsymbol{r}^{\prime}\right) \rho_{l k}\left(\boldsymbol{r}^{\prime}\right),
\end{aligned}
$$

and $\rho_{i j}(\boldsymbol{r})=(i / 2) \Phi_{i}^{\dagger}(\boldsymbol{r}) \tau^{z} \Phi_{j}(\boldsymbol{r})$ is the charge density associated with the pair of zero modes $\chi_{i}$ and $\chi_{j}$. We observe that at the neutrality point, when $\tilde{K}_{i j}=0$, the lowenergy effective Hamiltonian Eq. (2.10) coincides with the SYK model. Equations (2.11) and (2.12) allow us to calculate the relevant two- and four-fermion coupling constants from the knowledge of the Majorana wave functions in the noninteracting system. We carry out this program in Sec. IV for a specific physically relevant model system. Here, we finish by discussing some general properties of the Hamiltonian Eq. (2.10) that follow from symmetry considerations.

The reality condition Eq. (2.8) for the Majorana wave function implies the following spinor structure of $\Phi_{j}(\boldsymbol{r})$ in the Nambu space:

$$
\Phi_{j}=\left(\begin{array}{c}
\eta_{j} \\
i \sigma^{y} \eta_{j}^{*}
\end{array}\right)
$$

where $\eta_{j}(r)$ is a two-component complex spinor. We thus have

$$
\rho_{i j}=\frac{i}{2}\left(\eta_{i}^{\dagger} \eta_{j}-\text { c.c. }\right)=-\operatorname{Im}\left(\eta_{i}^{\dagger} \eta_{j}\right) .
$$

The charge density is thus purely real and antisymmetric under $i \leftrightarrow j$. In the simplest case, the $\Sigma$-breaking part of the Fu-Kane Hamiltonian will simply be $\delta H_{\mathrm{FK}}(\boldsymbol{r})=-\mu \tau^{z}$. In this situation, Eq. (2.11) implies that $\tilde{K}_{i j}=4 \mu \int d^{2} r \rho_{i j}(\boldsymbol{r})$. Thus, $\tilde{K}_{i j}$ is purely real and antisymmetric, as required for $\mathcal{H}_{\text {eff }}$ to be Hermitian.

Because of the anticommutation property Eq. (1.2) of the Majorana operators, it is clear that only the fully antisymmetric part of $\tilde{J}_{i j k l}$ contributes to the Hamiltonian Eq. (2.10). As we define in Eq. (2.12), $\tilde{J}_{i j k l}$ is already antisymmetric under $i \leftrightarrow j$ and $k \leftrightarrow l$ due to the antisymmetry $\rho_{i j}=-\rho_{j i}$. With this in mind we can rewrite the Hamiltonian Eq. (2.10) in a more convenient form:

$$
\mathcal{H}_{\mathrm{eff}}=i \sum_{i<j} K_{i j} \chi_{i} \chi_{j}+\sum_{i<j<k<l} J_{i j k l} \chi_{i} \chi_{j} \chi_{k} \chi_{l},
$$

with

$$
K_{i j}=\frac{1}{2}\left(\tilde{K}_{i j}-\tilde{K}_{j i}\right), \quad J_{i j k l}=\frac{1}{3}\left(\tilde{J}_{i j k l}-\tilde{J}_{i k j l}+\tilde{J}_{l i j k}\right)
$$

now fully antisymmetric. In the following, we are interested in situations where coupling constants are random and we characterize the coupling strengths by two parameters $K$ and $J$ defined by

$$
K^{2}=N \overline{K_{i j}^{2}}, \quad J^{2}=\frac{N^{3}}{3 !} \overline{J_{i j k l}^{2}}
$$


where the bar represents an ensemble average over randomness.

\section{Structure and statistics of the coupling constants $\boldsymbol{J}_{i j k l}$}

In order to approximate the SYK Hamiltonian, the coupling constants $J_{i j k l}$ we give in the previous section must behave as independent random variables. To assess this condition, we now discuss their structure and statistics. We make two reasonable assumptions: (i) the interaction potential in Eq. (2.12) is short ranged and well approximated by $V(\boldsymbol{r}) \simeq V_{0} \delta(\boldsymbol{r})$ and (ii) there exists a length scale $\zeta$ beyond which Majorana wave functions $\Phi_{j}(\boldsymbol{r})$ can be treated as random independent variables.

We coarse grain the Majorana wave functions on the grid with sites $\boldsymbol{r}_{n}$ and spacing $\sim \zeta$. This amounts to replacing $\eta_{j}(\boldsymbol{r}) \rightarrow \bar{\eta}_{j}\left(\boldsymbol{r}_{n}\right) / \zeta$ and $\int d^{2} r \rightarrow \zeta^{2} \sum_{n}$ in Eqs. (2.13) and (2.12). The discretized spinor wave functions then have the following structure on each site:

$$
\bar{\eta}_{j}\left(\boldsymbol{r}_{n}\right)=\left(\begin{array}{c}
\phi_{j}^{1}(n)+i \phi_{j}^{2}(n) \\
\phi_{j}^{3}(n)+i \phi_{j}^{4}(n)
\end{array}\right),
$$

where $\phi_{j}^{\alpha}(n)$ are real independent random variables with

$$
\overline{\phi_{i}^{\alpha}(n)}=0, \quad \overline{\phi_{i}^{\alpha}(n) \phi_{j}^{\beta}(n)}=\frac{1}{8 M_{s}} \delta_{i j} \delta^{\alpha \beta} .
$$

Here, $M_{s}=\pi R^{2} / \zeta^{2}$ is the total number of grid sites in the hole and the second equality follows from the normalization of $\Phi_{j}(\boldsymbol{r})$.

Combining Eqs. (2.12), (2.14), (2.16), and (2.18) it is possible to express the antisymmetrized coupling constants as

$$
J_{i j k l}=-\frac{V_{0}}{\zeta^{2}} \sum_{n=1}^{M_{s}} \epsilon_{\alpha \beta \mu \nu} \phi_{i}^{\alpha}(n) \phi_{j}^{\beta}(n) \phi_{k}^{\mu}(n) \phi_{l}^{\nu}(n),
$$

where $\epsilon_{\alpha \beta \mu \nu}$ is the totally antisymmetric tensor and summation over repeated indices is implied. For a general value of $M_{s}$, the many-body Hamiltonian defined by coupling constants Eq. (2.20) represents a variant of the original SYK model similar to models studied in Refs. $[14,16]$. As such, it might be amenable to the large- $N$ analysis using approaches described in those works. Here, we focus on the limit $M_{s} \gg N$, which works when the hole radius $R$ is large and the wave functions can be considered random on short scales $\zeta$. In this limit each $J_{i j k l}$ defined in Eq. (2.20) is given by a sum of a large number of random terms given by products of four random amplitudes $\phi_{j}^{\alpha}(n)$. The central limit theorem then assures us that $J$ 's will be random variables with a distribution approaching the Gaussian distribution irrespective of the detailed statistical properies of $\phi_{j}^{\alpha}(n)$. It is furthermore easy to show that

$$
\overline{J_{I}}=0, \quad \overline{J_{I} J_{J}}=\left(\frac{3 V_{0}}{8 \zeta^{2}}\right)^{2} \frac{1}{M_{s}^{3}} \delta_{I J}
$$

where the uppercase label represents a group of four indices $I=\{i j k l\}$. The coupling constants given by Eq. (2.20) are asymptotically independent with the higher-order correlators vanishing as higher powers of $M_{s}$, e.g., $\overline{J_{i j k l} J_{k l m n} J_{m n i j}} \sim M_{s}^{-5}$.

The above analysis suggests that under reasonable assumptions coupling constants defining the many-body Hamiltonian Eq. (2.15) can be considered independent random variables. When additionally $K_{i j}$ can be taken as negligible, we expect the Hamiltonian to approximate the SYK model. Building on the experience gained from Refs. $[14,16]$, we furthermore expect our Hamiltonian to describe an interesting non-Fermi-liquid phase even away from the limit when $J$ 's are independent variables. For instance, certain specific correlations present in $J$ 's are known to lead to a very interesting supersymmetric version of the SYK model [14] and a whole family of SYK-like models discussed in Ref. [16] .

Recent work [41] performed a mathematical analysis of deviations in $J$ 's from ideal random independent variables in a model qualitatively similar to ours. Here, we adopt a different approach and proceed by evaluating the effect of such deviations on the observable physical properties of the many-body model defined by the Hamiltonian Eq. (2.15). We find that coupling constants that follow from the giant vortex geometry indeed give rise to a phenomenology that is consistent with the SYK model.

\section{LARGE- $N$ SOLUTION AND THE CONFORMAL LIMIT}

When the number of Majorana fermions $N$ is large, the SYK model becomes analytically solvable in the low-energy limit. Specifically, the Euclidean-space timeordered propagator defined as

$$
G(\tau)=\left\langle\mathcal{T}_{\tau} \chi(\tau) \chi(0)\right\rangle
$$

can be expressed in the Matsubara frequency domain through the self-energy $\Sigma\left(\omega_{n}\right)$ as

$$
G\left(\omega_{n}\right)=\left[-i \omega_{n}-\Sigma\left(\omega_{n}\right)\right]^{-1} .
$$

Here, $G\left(\omega_{n}\right)=\int_{0}^{\beta} d \tau e^{i \omega_{n} \tau} G(\tau)$, and $\beta=1 / k_{B} T$ is the inverse temperature. At nonzero temperatures the propagator and the self-energy are defined for discrete Matsubara frequencies $\omega_{n}=\pi T(2 n+1)$, with $n$ integer and taking $k_{B}=1$ here and henceforth. Using the replica trick to average over disorder configurations, or alternately summing the leading diagrams in the $1 / N$ expansion, one obtains (see, for example, Ref. [7]) the following expression for the self-energy appropriate for the Hamiltonian Eq. (2.15): 


$$
\Sigma(\tau)=K^{2} G(\tau)+J^{2} G^{3}(\tau)
$$

For arbitrary given parameters $K, J$, and $\beta$ the selfconsistent equations (3.2) and (3.3) can be solved by numerical iteration. Analytical solutions are available in various limits and are reviewed below. In subsequent sections we compare these with numerical results based on the model described above.

\section{A. Free-fermion limit}

When $J=0$ the theory becomes noninteracting and an analytic solution to Eqs. (3.2) and (3.3) can be given for all temperatures. Specifically, the self-energy in Eq. (3.3) can be written in the frequency domain as $\Sigma\left(\omega_{n}\right)=K^{2} G\left(\omega_{n}\right)$ and substituted into Eq. (3.2). Solving for $G\left(\omega_{n}\right)$ then gives

$$
G_{f}\left(\omega_{n}\right)=\frac{2 i}{\omega_{n}+\operatorname{sgn}\left(\omega_{n}\right) \sqrt{\omega_{n}^{2}+4 K^{2}}} .
$$

This implies the high-frequency limit $G_{f}\left(\omega_{n}\right) \simeq i / \omega_{n}$ and the low-frequency limit $G_{f}\left(\omega_{n}\right) \simeq i / \operatorname{sgn}\left(\omega_{n}\right) K$.

It is useful to extract the single-particle spectral function from Eq. (3.4) defined as $A(\omega)=(1 / \pi) \operatorname{Im} G\left(\omega_{n} \rightarrow-i \omega+\delta\right)$, by analytically continuing from Matsubara to real frequencies to obtain the retarded propagator. We thus find

$$
A_{f}(\omega)=\frac{1}{\pi K} \operatorname{Re} \sqrt{1-\left(\frac{\omega}{2 K}\right)^{2}},
$$

the usual semicircle law. For this zero-dimensional system $A(\omega)$ coincides with the local density of states $D(\omega)$ averaged over all Majorana sites, which is experimentally measurable in a tunneling experiment. Specifically, the tunneling conductance $g(\omega)=(d I / d V)_{\omega=e V}$ is proportional to the local density of states $D(\omega)$.

\section{B. Conformal limit}

When $K=0$ and $T \ll J$ the system is strongly interacting but, nevertheless, an asymptotic solution of Eqs. (3.2) and (3.3) can be found by appealing to their approximate reparametrization invariance $[6,7]$ that becomes exact in the low-frequency limit when one can neglect the $-i \omega_{n}$ term in Eq. (3.2). The conformal limit solution reads

$$
G_{c}\left(\omega_{n}\right)=i \pi^{1 / 4} \frac{\operatorname{sgn}\left(\omega_{n}\right)}{\sqrt{J\left|\omega_{n}\right|}}
$$

and the corresponding spectral function is

$$
A_{c}(\omega)=\frac{1}{\sqrt{2} \pi^{3 / 4}} \frac{1}{\sqrt{J|\omega|}}
$$

These expressions are valid for $|\omega| \ll J$ and must cross over to the $1 / \omega$ behavior at large frequencies.
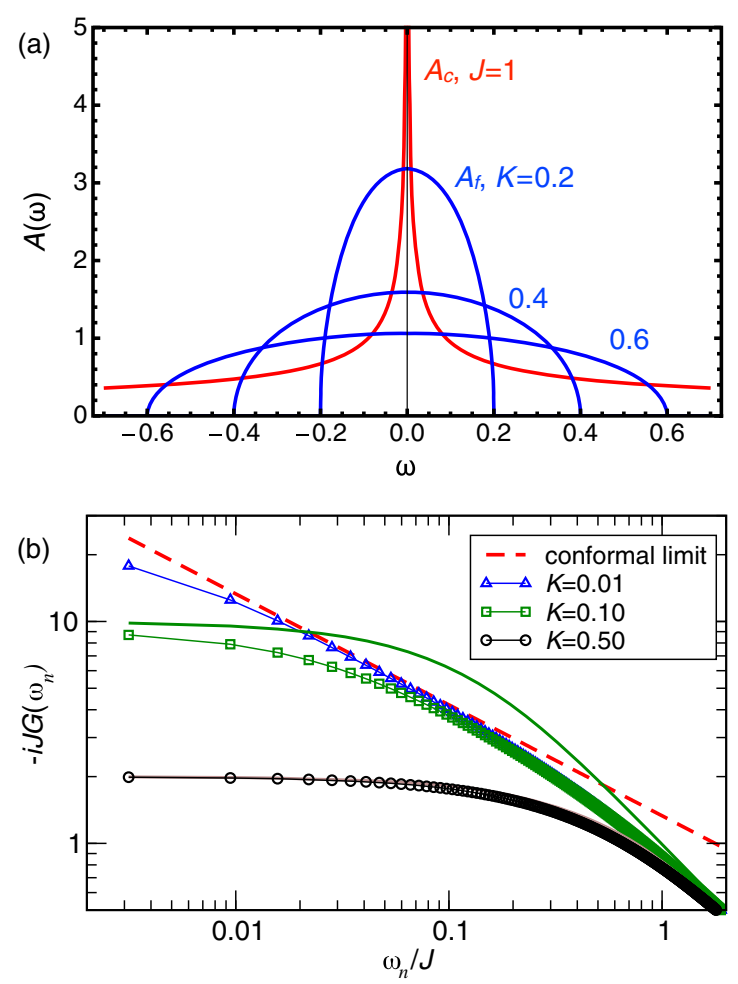

FIG. 2. (a) Spectral functions, measurable in a tunneling experiment, in the conformal (strongly interacting) limit (red lines) and free-fermion limit (blue lines). (b) Numerically evaluated large- $N$ Matsubara Green's functions for $J=1.0$, $T=0.001$, and different values of $K$. Red dashed line shows the conformal limit behavior Eq. (3.7) while the thick green and brown lines correspond to free-fermion result Eq. (3.4) with $K=0.1$ and 0.5 , respectively.

It is important to note that the low-frequency behaviors of $A_{f}$ and $A_{c}$ are quite different with the former saturating at $1 / \pi K$ and the latter divergent. Thus, it should be possible to distinguish the free-fermion and the interaction-dominated behaviors, illustrated in Fig. 2(a), by performing a tunneling experiment. We discuss the measurement in more detail in Sec. VI.

\section{Crossover region}

When both $K$ and $J$ are nonzero, as is the case in a typical experimental setup, analytical solutions are not available, but one can still understand the behavior of the system from approximate analytical considerations and numerical solutions. Let us focus on the $T=0$ limit and study the effect of $K$ and $J$ on the self-energy $\Sigma\left(\omega_{n}\right)$ in Eq. (3.3). To this end, it is useful to consider the propagators $G_{f}$ and $G_{c}$ in the imaginary time domain. For long times $\tau$, one obtains

$G_{f}(\tau)=\frac{1}{\pi K} \frac{\operatorname{sgn}(\tau)}{|\tau|}, \quad G_{c}(\tau)=\frac{1}{\pi^{1 / 4} \sqrt{2 J}} \frac{\operatorname{sgn}(\tau)}{|\tau|^{1 / 2}}$. 
Consider the $K=0$ limit and then slowly turn $K$ on. Initially, $G_{c}(\tau)$ is a valid solution. However, for any nonzero $K$ it is clear that the first term on the right-hand side of Eq. (3.3) will dominate at sufficiently long times $\tau>\tau_{*}$. At such long times one then expects a crossover to the behavior resembling the free-fermion propagator $G_{f}(\tau)$. The corresponding crossover time $\tau_{*}$ can be estimated by equating the two terms on the right-hand side of Eq. (3.3), $K^{2} G_{f}\left(\tau_{*}\right)=J^{2} G_{c}^{3}\left(\tau_{*}\right)$, which gives

$$
\tau_{*}=\frac{\sqrt{\pi}}{8} \frac{J}{K^{2}},
$$

and the corresponding crossover frequency

$$
\omega_{*}=\frac{2 \pi}{\tau_{*}}=16 \sqrt{\pi} \frac{K^{2}}{J} .
$$

We thus expect the spectral function to behave as indicated in Eq. (3.7) for $\omega_{*}<\omega \ll J$ with the divergence at small $\omega$ cut off below $\omega_{*}$ and saturate to $\sim 1 / \pi K$.

To confirm the above behavior, we solved Eqs. (3.2) and (3.3) numerically. We find it most convenient to work with Matsubara Green's functions at very low but nonzero temperatures. To this end, we rewrite Eq. (3.3) in Matsubara frequency domain where the last term becomes a convolution and substitute the self-energy into Eq. (3.2). We obtain a single equation,

$$
G_{n}^{-1}=-i \omega_{n}-K^{2} G_{n}-J^{2} T^{2} \sum_{k, l} G_{k} G_{l} G_{n-k-l}
$$

for $G_{n} \equiv G\left(\omega_{n}\right)$ that must be solved self-consistently. Results obtained by iterating Eq. (3.11) are displayed in Fig. 2(b). For very small $K=0.01 J$, we observe that numerically calculated $G\left(\omega_{n}\right)$ coincides with the conformal limit for a range of frequencies consistent with our discussion above. For $K=0.1 J$, this range becomes smaller and completely disappears for $K=0.5 \mathrm{~J}$.

We conclude that for any nonzero $K$ the ultimate lowenergy behavior is controlled by the free-fermion fixed point, as expected on general grounds. Nevertheless, when $K$ is sufficiently small in comparison to $J$, there can be a significant range of energies in which the physics is dominated by the SYK fixed point. At low temperatures the corresponding range of frequencies is given by

$$
16 \sqrt{\pi} \frac{K^{2}}{J}<\omega \ll J
$$

In this range we expect the spectral function to obey the conformal scaling form given by Eq. (3.7). A tunneling experiment in this regime should therefore reveal the SYK behavior of the underlying strongly interacting system.

\section{NUMERICAL RESULTS: THE UNDERLYING NONINTERACTING SYSTEM}

In this section, we provide support for the ideas we present above by performing extensive numerical simulation and modeling of the system we describe in Sec. II. We start by formulating a lattice model for the surface of a TI in contact with a superconductor. We then find the wave functions of the Majorana zero modes by numerically diagonalizing the corresponding Bogoliubov-de Gennes (BdG) Hamiltonian for the geometry depicted in Fig. 1 with $N$ flux quanta threading the hole. In the following section, using Eqs. (2.11) and (2.12), we calculate the coupling constants $K_{i j}$ and $J_{i j k l}$, which we then use to construct and diagonalize the many-body interacting Hamiltonian Eq. (2.15) for $N$ up to 32. The resulting many-body spectra and eigenvectors are used to calculate various physical quantities (entropy, specific heat, two- and four-point correlators), which are then compared to the results previously obtained for the SYK model with random independent couplings.

\section{A. Lattice model for the TI surface}

A surface of a 3D TI is characterized by an odd number of massless Dirac fermions protected by time-reversal symmetry $\Theta$. The well-known Nielsen-Ninomyia theorem $[52,53]$ assures us that, as a matter of principle, it is impossible to construct a purely $2 \mathrm{D}$, $\Theta$-invariant lattice model with an odd number of massless Dirac fermions. This fact causes a severe problem for numerical approaches to 3D TIs because one is forced to perform an expensive simulation of the $3 \mathrm{D}$ bulk to describe the anomalous $2 \mathrm{D}$ surface. A workaround has been proposed [54] that circumvents the Nielsen-Ninomyia theorem by simulating a pair of TI surfaces with a total even number of Dirac fermions. This approach enables efficient numerical simulations in a quasi-2D geometry while fully respecting $\Theta$.

Here, because the physical time-reversal symmetry is ultimately broken by the presence of vortices and is therefore not instrumental, we opt for an even simpler model which breaks $\Theta$ from the outset but nevertheless captures all the essential physics of the TI-SC interface. We start from the following momentum-space normal-state Hamiltonian defined on a simple 2D square lattice:

$$
h_{0}(\boldsymbol{k})=\lambda\left(\sigma^{x} \sin k_{x}+\sigma^{y} \sin k_{y}\right)+\sigma^{z} M_{k}-\mu,
$$

with $M_{k}=m\left[\left(2-\cos k_{x}-\cos k_{y}\right)-\frac{1}{4}\left(2-\cos 2 k_{x}-\cos 2 k_{y}\right)\right]$. Here, $\sigma$ are Pauli matrices in spin space and $\lambda, m$ are model parameters. The term proportional to $\lambda$ respects $\Theta$ and gives four massless Dirac fermions in accordance with the Nielsen-Ninomyia theorem. The $M_{k}$ term breaks $\Theta$ and has the effect of gapping out all the Dirac fermions except the one located at $\Gamma=(0,0)$. The resulting energy spectrum, 


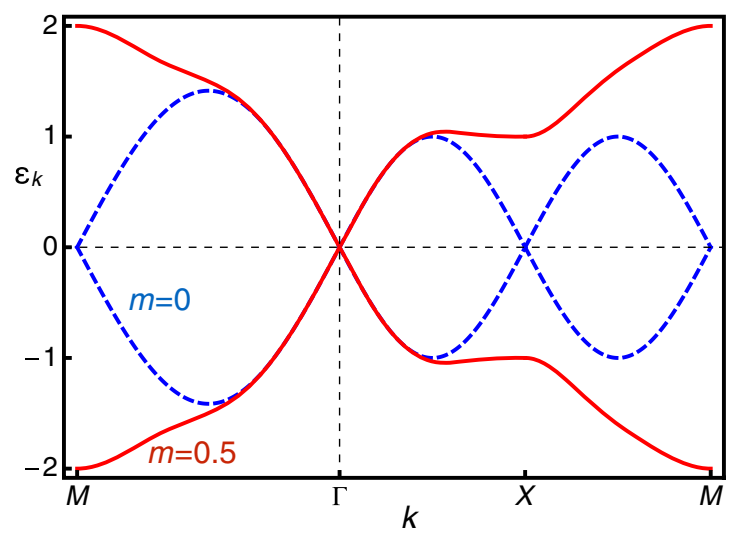

FIG. 3. Band structure (4.2) of the lattice model Eq. (4.1) for $\lambda=1$ and $m=0$ (blue dashed) and $m=0.5$ (red solid line). $X$ and $M$ denote the $(0, \pi)$ and $(\pi, \pi)$ points of the Brillouin zone, respectively.

$$
\varepsilon(\boldsymbol{k})= \pm \sqrt{\lambda^{2}\left(\sin ^{2} k_{x}+\sin ^{2} k_{y}\right)+M_{\boldsymbol{k}}^{2}}-\mu
$$

is depicted in Fig. 3. In the vicinity of the $\Gamma$ point, we observe a linearly dispersing spectrum characteristic of a TI surface state. It is to be noted that for small $|\boldsymbol{k}|$ we have $M_{k} \simeq \frac{1}{8} m k^{4}$, so the amount of $\Theta$ breaking can be considered small in the physically important part of the momentum space near the $\Gamma$ point.

Proximity-induced superconducting order is implemented by constructing the BdG Hamiltonian:

$$
H_{\mathrm{BdG}}(\boldsymbol{k})=\left(\begin{array}{cc}
h_{0}(\boldsymbol{k}) & \Delta \\
\Delta^{*} & -\sigma^{y} h_{0}^{*}(-\boldsymbol{k}) \sigma^{y}
\end{array}\right) .
$$

Writing $H_{\mathrm{BdG}}$ in terms of $\sigma$ and $\tau$ matrices it can be easily checked that it respects the particle-hole symmetry $\Xi$ we define in Sec. II. A. The $\mu$ and $M_{k}$ terms both break the fictitious time reversal $\Sigma$ that protects the Majorana zero modes in our setup. As before, $\mu$ must be tuned to zero to achieve protection. On the other hand, it is crucial to remember that $M_{k}$ has been introduced only to circumvent the Nielsen-Ninomyia theorem and allow us to efficiently simulate a single two-dimensional Dirac fermion on the lattice. Breaking of $\Sigma$ by $M_{k}$ is therefore not a concern in the experimental setup: in a real TI tuned to the neutrality point, $\Sigma$ is unbroken. Expanding $H_{\mathrm{BdG}}(\boldsymbol{k})$ in the vicinity of $\Gamma$ to leading order in small $\boldsymbol{k}$ we recover the Fu-Kane Hamiltonian $H_{\mathrm{FK}}$ defined in Eq. (2.2). We thus conclude that at low energies our lattice model indeed describes the TI-SC interface and should exhibit the desired phenomenology, including Majorana zero modes bound to vortices. We show that this is indeed the case. The only repercussion that follows from the weakly broken $\Sigma$ (present in the higher-order terms in the above expansion) is a very small splitting of the zero-mode energies that has no significant effect on our results.

\section{B. Solution in the giant vortex geometry}

To study the nonuniform system with magnetic field and vortices, we must write the Hamiltonian in the position space. The normal-state piece Eq. (4.1) is most conveniently written in second-quantized form as

$$
\begin{aligned}
\mathcal{H}_{0}= & i \lambda \sum_{\boldsymbol{r}, \alpha}\left(\psi_{\boldsymbol{r}}^{\dagger} \sigma^{\alpha} \psi_{\boldsymbol{r}+\alpha}-\text { H.c. }\right)+\sum_{\boldsymbol{r}} \psi_{\boldsymbol{r}}^{\dagger}\left(\frac{3}{2} m \sigma^{z}-\mu\right) \psi_{\boldsymbol{r}} \\
& -\frac{m}{8} \sum_{\boldsymbol{r}, \alpha}\left(4 \psi_{\boldsymbol{r}}^{\dagger} \sigma^{z} \psi_{\boldsymbol{r}+\alpha}-\psi_{\boldsymbol{r}}^{\dagger} \sigma^{z} \psi_{\boldsymbol{r}+2 \alpha}+\text { H.c. }\right),
\end{aligned}
$$

where we define on each lattice site $\boldsymbol{r}$ a two-component spinor $\psi_{\boldsymbol{r}}=\left(c_{\boldsymbol{r} \uparrow}, c_{\boldsymbol{r} \downarrow}\right)^{T}$ and $\alpha=x, y$. The magnetic field is included through the standard Peierls substitution, which replaces tunneling amplitudes on all bonds according to $\psi_{\boldsymbol{r}}^{\dagger} \psi_{\boldsymbol{r}+\alpha} \rightarrow \psi_{\boldsymbol{r}}^{\dagger} \psi_{\boldsymbol{r}+\alpha} \exp \left\{-i[e /(\hbar c)] \int_{\boldsymbol{r}}^{\boldsymbol{r}+\alpha} d \boldsymbol{l} \cdot \boldsymbol{A}\right\}$. The full second-quantized BdG Hamiltonian then reads

$$
\mathcal{H}_{\mathrm{BdG}}=\mathcal{H}_{0}+\sum_{\boldsymbol{r}}\left(\Delta_{\boldsymbol{r}} c_{\boldsymbol{r} \uparrow}^{\dagger} c_{\boldsymbol{r} \downarrow}^{\dagger}+\text { H.c. }\right)
$$

where $\Delta_{r}$ is the pair potential on site $\boldsymbol{r}$, which takes the form indicated in Eq. (2.3). In accord with our discussion in the previous section, $\mathcal{H}_{\mathrm{BdG}}$ given in Eq. (4.5) represents a version of the Fu-Kane Hamiltonian Eq. (2.1) regularized on a square lattice. This lattice model is suitable for numerical calculations and we expect it to reproduce all the low-energy features of the Fu-Kane Hamiltonian. In particular, we show shortly that it yields $N$ Majorana zero modes mandated by the Jackiw-Rossi-Weinberg index theorem that are of central importance for the SYK model.

It is most convenient to solve the problem defined by Hamiltonian Eq. (4.5) on a lattice with $L \times L$ sites and periodic boundary conditions which ensure that no spurious edge states exist at low energies in addition to the expected $N$ Majorana zero modes bound to the hole. To implement periodic boundary conditions, it is useful to perform a singular gauge transformation,

$$
\psi_{\boldsymbol{r}} \rightarrow e^{i N \varphi / 2} \psi_{\boldsymbol{r}}
$$

which has the effect of removing the phase winding from $\Delta_{r}$ and changing the Peierls phase factors to $\exp \left(i \int_{\boldsymbol{r}}^{\boldsymbol{r}+\alpha} d \boldsymbol{l} \cdot \boldsymbol{\Omega}\right)$, with

$$
\boldsymbol{\Omega}=\frac{1}{2}\left(N \nabla \varphi-\frac{2 e}{\hbar c} \boldsymbol{A}\right) .
$$

We note that $N$ must be even because only for integer number of fundamental flux quanta $h c / e=2 \Phi_{0}$ in the system can one impose periodic boundary conditions. For $N$ even, the transformation Eq. (4.6) is single valued and the issue of branch cuts that renders the analogous problem with singly quantized vortices [55,56] more complicated does not arise here. After the transformation the total effective flux seen by the electrons $\int d S(\boldsymbol{\nabla} \times \boldsymbol{\Omega})_{z}$ vanishes 
and numerical diagonalization of the transformed Hamiltonian Eq. (4.5) with periodic boundary conditions becomes straightforward.

As a practical matter, it is easiest to define a regularshaped hole and introduce disorder through a replacement:

$$
\left(\mu, \lambda, \Delta_{r}\right) \rightarrow\left(\mu, \lambda, \Delta_{r}\right)+\left(\delta \mu_{r}, \delta \lambda_{r}, \delta \Delta_{r}\right) .
$$

Here, $\left(\delta \mu_{r}, \delta \Delta_{r}, \delta \lambda_{r}\right)$ are independent random variables uniformly distributed in the interval $\left(-w_{\mu} / 2, w_{\mu} / 2\right)$ for $\delta \mu_{r}$ and similarly for $\delta \Delta_{r}$ and $\delta \lambda_{r}$. We choose a stadiumshaped hole sketched in Fig. 4(a), which is known to support classically chaotic trajectories [39,40]. In our quantum simulation we find that much smaller disorder strength is required to achieve sufficiently random Majorana wave functions for a stadium-shaped hole than, e.g., with circular hole. We furthermore choose magnetic field $B$ to be uniform inside the radius $R_{B}$ that contains the hole and zero otherwise. We find that our results are insensitive to the detailed distribution of $B$ as long as the total flux remains $N \Phi_{0}$ and is centered around the hole (we test various radii $R_{B}$ as well as a Gaussian profile).

Typical results of the numerical simulations we describe above are displayed in Fig. 4. In Fig. 4(b), we observe the behavior of the energy eigenvalues $E_{n}$ of $\mathcal{H}_{\mathrm{BdG}}$. For zero magnetic flux, there are several states inside the SC gap (Andreev states bound to the hole) but no zero modes. For $N=24$, these are converted into 24 zero modes required by the Jackiw-Rossi-Weinberg index theorem. For $\mu=w_{\mu}=0$ used in the simulation, their energies are very close to zero $\left(\sim 10^{-4} \lambda\right)$, where the small residual splitting is attributable

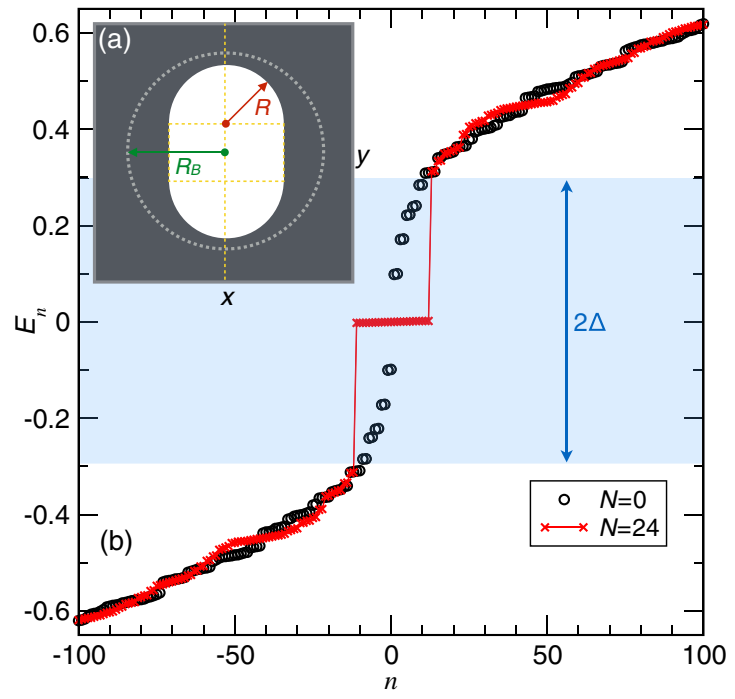

to the fact that $\Sigma$ symmetry is weakly broken in our lattice simulation by the $M_{k}$ term. For nonzero $\mu$ or $w_{\mu}$, the energy splitting increases in proportion to these $\Sigma$-breaking perturbations. In the following, we include these terms in $\delta \mathcal{H}_{\mathrm{FK}}$ and incorporate them in our many-body calculation via $K_{i j}$ terms given by Eq. (2.11).

Figures 4(c)-4(f) show examples of zero-mode wave function amplitudes $\left|\Phi_{j}(\boldsymbol{r})\right|^{2}$. The wave functions are shown to exhibit random spatial structure, which depends sensitively on the specific disorder potential realization. Importantly, all the zero-mode wave functions are localized in the same region of space defined by the hole and its immediate vicinity. One therefore expects Eq. (2.12) to produce strong random couplings $J_{i j k l}$ connecting all zero modes $\chi_{j}$ once the interactions are included.

\section{NUMERICAL RESULTS: THE MANY-BODY SYK PROBLEM}

Having obtained the zero-mode wave functions it is straightforward to calculate couplings $K_{i j}$ and $J_{i j k l}$ from Eqs. (2.11) and (2.12) and construct the many-body SYK Hamiltonian Eq. (2.15). In the following, we assume that the system has been tuned to its global neutrality point $\mu=0$ and include in $\delta \mathcal{H}_{\mathrm{FK}}$ only the random component of the on-site potential $\delta \mu_{r}$. For the interaction term we consider the screened Coulomb potential defined as

$$
V(r)=\frac{2 \pi e^{2}}{\epsilon} \frac{e^{-r / \lambda_{\mathrm{TF}}}}{r},
$$
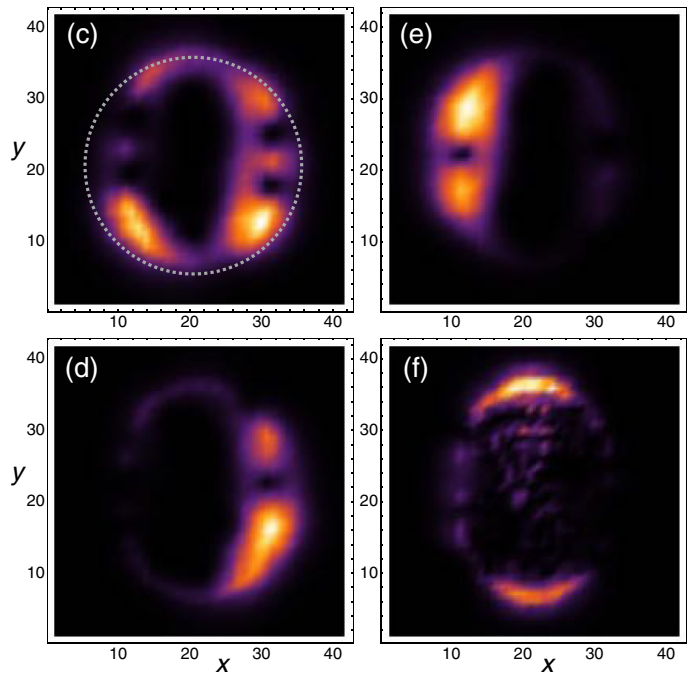

FIG. 4. Numerical simulations of the BdG Hamiltonian Eq. (4.5). (a) Stadium-shaped hole geometry employed in the simulations. $R$ parametrizes the hole size whereas $R_{B}$ denotes the radius inside which the magnetic field is nonzero. (b) Energy levels $E_{n}$ of the BdG Hamiltonian Eq. (4.5) calculated for $N=0$ and $N=24$. Energies have been sorted in ascending order and plotted as a function of their integer index $n$. The shaded band represents the SC gap region. (c)-(f) Density plots of the typical zero-mode wave function amplitudes for $N=24$. The dashed circle in (c) has radius $R_{B}$. The following parameters are used to obtain these results: $\lambda=1, m=0.5, \Delta_{0}=0.3$, $\mu=w_{\mu}=0, w_{\lambda}=w_{\Delta}=0.1, L=42, R=10$, and $R_{B}=15$. 
where $\epsilon$ is the dielectric constant and $\lambda_{\mathrm{TF}}$ denotes the Thomas-Fermi screening length. We furthermore assume that $\lambda_{\mathrm{TF}}$ is short, so that in the lattice model the interaction is essentially on site. The expression for $\tilde{J}_{i j k l}$ then simplifies to

$$
\tilde{J}_{i j k l} \simeq 12 V_{0} \int d^{2} r \rho_{i j}(\boldsymbol{r}) \rho_{l k}(\boldsymbol{r})
$$

with $V_{0}=\int d^{2} r V(r)=2 \pi e^{2} \lambda_{\mathrm{TF}} / \epsilon$. Coupling constants $K_{i j}$ and $J_{i j k l}$ are easy to evaluate using Eq. (2.16) and the Majorana wave functions $\Phi_{j}(\boldsymbol{r})$ obtained in the previous section. To facilitate comparisons with the existing literature, we quantify the average strength of these terms using parameters $K$ and $J$ defined in Eq. (2.17). Specifically, we adjust $w_{\mu}$ and $V_{0}$ to obtain the desired values of $K$ and $J$. In the following section, we connect these values to the parameters expected in realistic physical systems.

\section{A. Thermodynamic properties and many-body level statistics}

Once the coupling constants $K_{i j}$ and $J_{i j k l}$ are determined as described above, one can construct a matrix representation of the many-body Majorana Hamiltonian Eq. (2.15) and find its energy eigenvalues $E_{n}$ by exact numerical diagonalization. From the knowledge of the energy levels, it is straightforward to calculate any thermodynamic property. In Fig. 5, we display the thermal entropy $S(T)$ and the heat capacity $C_{V}(T)$. These are calculated from

$$
S=\frac{\langle E\rangle-F}{T}, \quad C_{V}=\frac{\left\langle E^{2}\right\rangle-\langle E\rangle^{2}}{T},
$$

where $\left\langle E^{\alpha}\right\rangle=(1 / Z) \sum_{n} E_{n}^{\alpha} e^{-E_{n} / T}, F=-T \ln Z$ is the free energy, and $Z=\sum_{n} e^{-E_{n} / T}$ the partition function.

The entropy per particle is seen to saturate at high temperature to $S_{\infty} / N=\frac{1}{2} \ln 2 \simeq 0.3465$, as expected for a system of $N$ Majorana fermions. The behavior of $S(T)$ calculated for the giant vortex system is qualitatively similar to that obtained from the SYK model with random independent couplings. The small deviations that exist are clearly becoming smaller as $N$ grows, suggesting that they vanish in the thermodynamic limit. Nonzero two-body coupling $K$ is seen to modify the entropy slightly at low temperature. For large $N$ and $K=0$, the entropy per particle is expected to attain a nonzero value $\sim 0.24$ as $T \rightarrow$ 0 due to the extensive near ground-state degeneracy of the SYK model. Our largest system is not large enough to show this behavior (in agreement with previous numerical results) although Fig. 5(a) correctly captures the expected suppression of the low- $T$ entropy in the presence of twobody couplings, which tend to remove the extensive ground-state degeneracy.
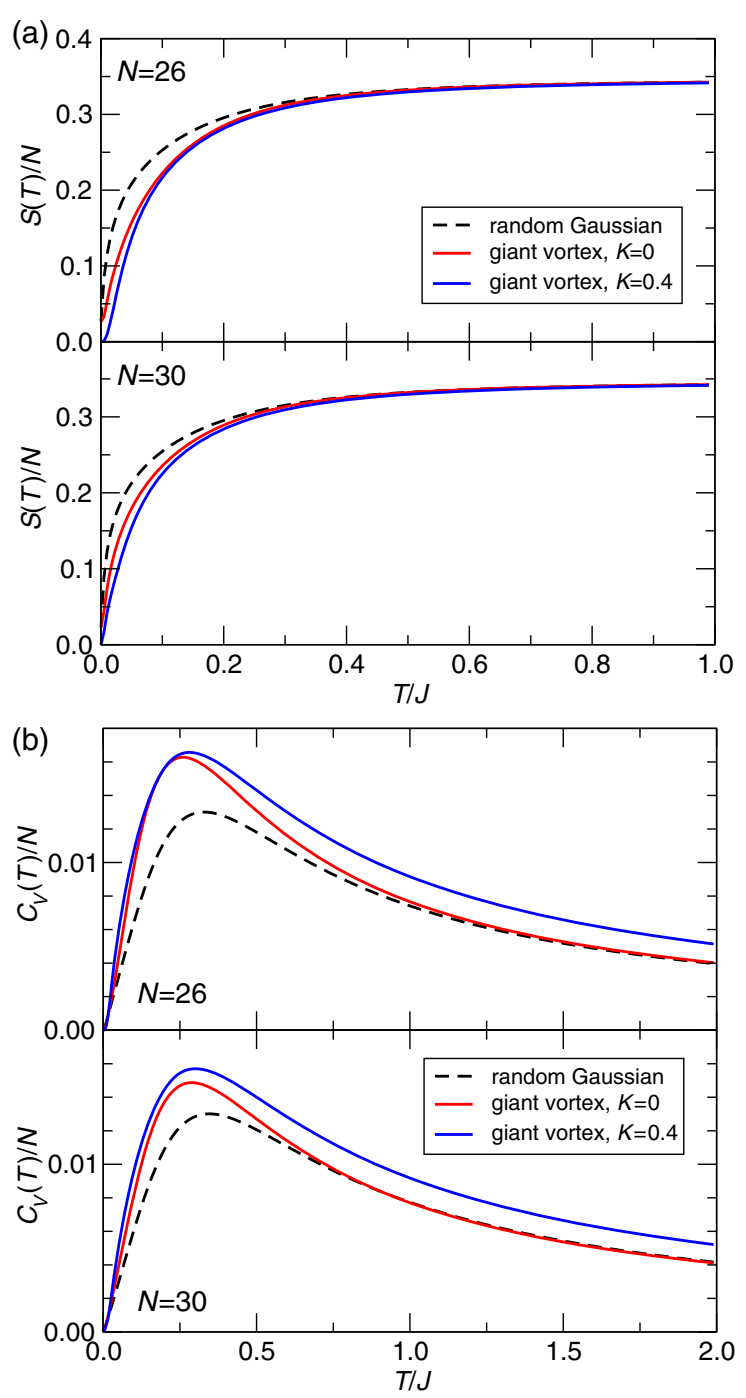

FIG. 5. Thermodynamic properties of the many-body Hamiltonian Eq. (2.15). (a) Thermal entropy per particle and (b) heat capacity per particle. Dashed lines show the expected behavior for the SYK model with random independent couplings, solid lines show results for the couplings obtained from the giant vortex system. In all panels the same parameters are used as in Fig. 4 with $V_{0}$ adjusted so that $J=1$.

The heat capacity $C_{V}(T)$, displayed in Fig. 5(b), likewise behaves as expected for the SYK model with random independent couplings with small deviations becoming negligible in the large- $N$ limit. $C_{V}(T)$ is, in principle, measurable, and we can see from Fig. 5(b) that its high-temperature behavior could be used to gauge the relative strength of two- and four-fermion terms in the system.

As discussed in Refs. [12,13], many-body level statistics provides a sensitive diagnostic for the SYK physics encoded in the Hamiltonian Eq. (2.15). To apply this analysis to our results, we arrange the many-body energy levels in ascending order $E_{1}<E_{2}<\cdots$ and form ratios between the successive energy spacings: 
TABLE I. Gaussian ensembles for even $N$.

\begin{tabular}{lcccc}
\hline \hline$N(\bmod 8)$ & 0 & 2 & 4 & 6 \\
\hline Level stat. & GOE & GUE & GSE & GUE \\
$\beta$ & 1 & 2 & 4 & 2 \\
$Z$ & $\frac{8}{27}$ & $4 \pi /(81 \sqrt{3})$ & $4 \pi /(729 \sqrt{3})$ & $4 \pi /(81 \sqrt{3})$ \\
\hline \hline
\end{tabular}

$$
r_{n}=\frac{E_{n+1}-E_{n}}{E_{n+2}-E_{n+1}} .
$$

According to Refs. $[12,13]$, the SYK Hamiltonian can be constructed as a symmetric matrix in the Clifford algebra $\mathcal{C} \ell_{0, N-1}$ whose Bott periodicity gives rise to a $Z_{8}$ classification with topological index $\nu=N \bmod 8$. As a result, statistical distributions of the ratios $P(r)$ cycle through Wigner-Dyson random matrix ensembles with $Z_{8}$ periodicity as a function of $N$. Specifically, Gaussian orthogonal (GOE), Gaussian unitary (GUE), and Gaussian symplectic (GSE) ensembles occur with distributions given by the "Wigner surmise,"

$$
P(r)=\frac{1}{Z} \frac{\left(r+r^{2}\right)^{\beta}}{\left(1+r+r^{2}\right)^{1+3 \beta / 2}},
$$

and parameters $Z$ and $\beta$ summarized in Table I for even $N$ relevant to our system. As emphasized in Ref. [13], the level spacing analysis must be performed separately in the two-fermion parity sectors of the Hamiltonian Eq. (2.15).

Figure 6 shows statistical distributions of the ratios $r_{n}$ computed for $N=24,26,28,30$, and 32 in our system. For the sake of clarity $P(\ln r)$ is plotted along with the anticipated distributions for GOE, GUE, and GSE given in Eq. (5.5). Unambiguous agreement with the pattern indicated in Table I is observed, lending further support to the notion that our proposed system realizes the SYK model. We check that the $Z_{8}$ periodic pattern persists for all $N$ down to 16. Additionally, the above results should be contrasted with the level statistics in the noninteracting case $J=0, K=1$ displayed in the bottom row of Fig. 6 . In the absence of interactions, $Z_{8}$ periodicity is absent and the distribution of the ratio $r_{n}$ follows Poisson level statistics,

$$
P(r)=\frac{1}{(1+r)^{2}},
$$

for all $N$. It is to be noted that no adjustable parameters are employed in the level-statistics analysis we present above.

\section{B. Green's function}

Computing the Green function of the model is perhaps the most straightforward way of comparing the behavior of the system at finite $N$ to the large- $N$ limit solutions we discuss in Sec. III. At the same time, computation of propagators is numerically more costly because in addition to many-body energy levels, one requires the corresponding eigenstates. We compute the on-site retarded Green function defined as

$$
G_{i}^{R}\left(t-t^{\prime}\right)=-i \theta\left(t-t^{\prime}\right)\left\langle\left\{\chi_{i}(t), \chi_{i}\left(t^{\prime}\right)\right\}\right\rangle .
$$

Fourier transforming and using Lehmann representation in terms of the eigenstates $|n\rangle$ of the many-body Hamiltonian Eq. (2.15), one obtains, at $T=0$,
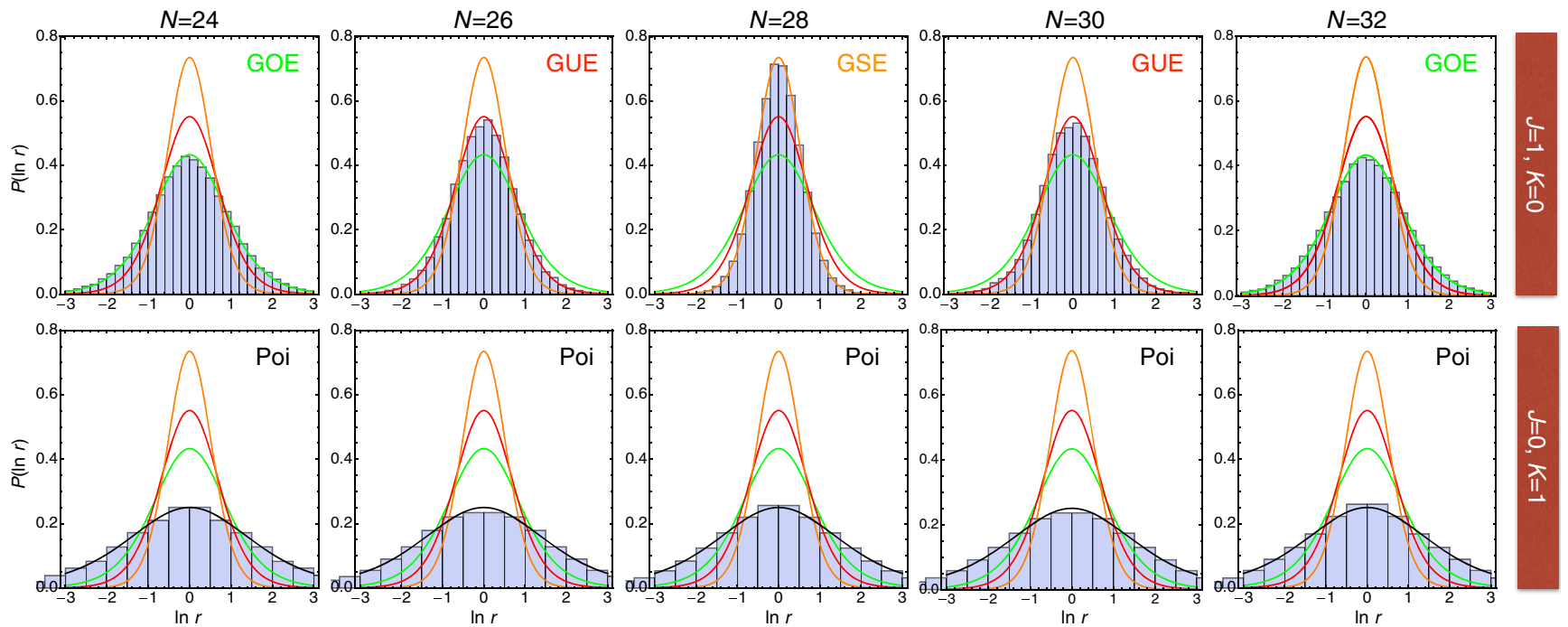

FIG. 6. Level statistics analysis. Top row: Histograms of $\ln r_{n}$ obtained from the energy levels of the SYK Hamiltonian Eq. (2.15) with coupling constants taken from the giant vortex model with $V_{0}$ and $w_{\mu}$ adjusted so that $J=1$ and $K=0$. Solid lines indicate the expected distributions GOE (green), GUE (red), and GSE (orange) specified in Eq. (5.5). Bottom row: Results for the noninteracting case $J=0$, $K=1$. Black solid line represents the Poisson distribution Eq. (5.6). Histograms in all panels are averaged over eight independent realizations of disorder except for $N=24$, where 16 realizations are employed to obtain satisfactory statistics, and $N=32$, for which a single realization is used. 
$G_{i}^{R}(\omega)=\sum_{n}\left[\frac{\left|\left\langle n\left|\chi_{i}\right| 0\right\rangle\right|^{2}}{\omega+E_{0}-E_{n}+i \delta}+\left(E_{0} \leftrightarrow E_{n}\right)\right]$,

where $\delta$ is a positive infinitesimal. From Eq. (5.8), the spectral function $A_{i}(\omega)=(1 / \pi) \operatorname{Im} G_{i}^{R}(\omega)$ is readily extracted.

In Fig. 7, we display the spectral function $A(\omega)=$ $(1 / N) \sum_{i} A_{i}(\omega)$ averaged over all Majorana zero modes. Physically this corresponds to a tunneling experiment with a large probe that allows for tunneling into all sites inside the hole. In agreement with the existing numerical results on the complex fermion version of the SYK model [57], we find that for system sizes we can numerically access (up to $N=30$ ), the conformal limit is approached only in a narrow interval of frequencies. In the low-frequency limit, numerical results approach a constant value instead of the the $\sim 1 / \sqrt{\omega}$ divergence expected in the large- $N$ limit. The dependence on $N$ is very weak, with the larger values showing reduced statistical fluctuations but otherwise qualitatively similar behavior. To convincingly demonstrate the conformal scaling of the Green function at the lowest frequencies, numerical calculations using larger values of $N$ would be necessary. Unfortunately, these are currently out of reach for the exact diagonalization method due to the

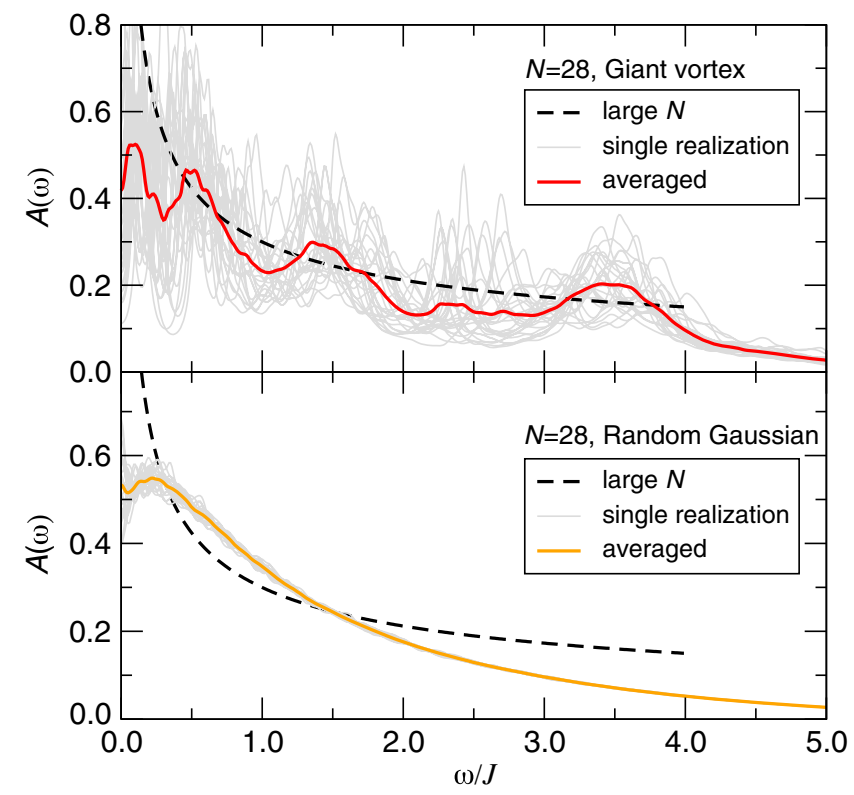

FIG. 7. Spectral function $A(\omega)$ computed at zero temperature for coupling constants $J_{i j k l}$ obtained from the giant vortex calculation (top) and taken from the Gaussian distribution (bottom). Thin gray lines represent individual disorder realizations corresponding to a physical measurement in a system with quenched disorder. Thick lines reflect the average over 25 independent disorder realizations. Dashed lines represent the expected low-frequency behavior in the large- $N$ conformal limit Eq. (3.7). All parameters are as in Fig. 4 with $J=1, K=0$ and broadening $\delta=0.04$ in Eq. (5.8). exponential growth of the Hamiltonian matrix size with $N$. More sophisticated numerical techniques, such as the quantum Monte Carlo method, could possibly reach larger system sizes.

Spectral functions calculated for the giant vortex setup exhibit larger statistical fluctuations compared to those computed with random Gaussian coupling constants $J_{i j k l}$ but are qualitatively similar when averaged over independent disorder realizations. Therefore, we conclude that the Green function behavior at finite $N$ supports the notion that our proposed system realizes the SYK model.

\section{Out-of-time-order correlators and scrambling}

Scrambling of quantum information-a process in which quantum information deposited into the system locally gets distributed among all its degrees of freedom-is central to the conjectured duality between the SYK model and $\mathrm{AdS}_{2}$ Einstein gravity. Black holes are thought to scramble with the maximum possible efficiency: they exhibit quantum chaos. For a quantum theory to be the holographic dual of a black hole, its dynamics must exhibit similar fast scrambling behavior.

The out-of-time-order correlator (OTOC), defined in our system as

$$
F_{i j}(t)=\left\langle\chi_{j}(t) \chi_{i}(0) \chi_{j}(t) \chi_{i}(0)\right\rangle,
$$

allows us to quantify the quantum chaotic behavior. For black holes in Einstein gravity scrambling occurs exponentially fast with $1-F(t) \sim e^{\lambda_{L} t} / N$, where the decay rate is given by the Lyapunov exponent $\lambda_{L}=2 \pi T$ [9]. Similarly, for the SYK model in the large- $N$ limit, one expects [6,7]

$$
1-F(t) \sim \frac{J}{N T} e^{\lambda_{L} t}
$$

Previous works [10,57] gave numerical evaluations of $F(t)$ in the SYK model for $N$ up to 14 but found these system sizes to be too small to clearly show the expected $J$-independent Lyapunov exponent. Here, we numerically evaluate OTOC for $N$ up to 22 and show that coupling constants obtained from the giant vortex geometry give qualitatively the same behavior as those for random independent coupling constants. Our results are summarized in Fig. 8, where we compute the on-site OTOC $F_{i i}(t)$ averaged over all sites.

For $J=1$, the OTOC is seen to rapidly decay to zero, consistent with previous works on the SYK model $[10,57]$. The rate of decay is controlled by $J$ : as in Refs. [10,57], we find that $N=22$ is not large enough to observe the theoretically predicted $J$-independent Lyapunov exponent controlled by temperature, even when $J \gg T$. In addition, we observe that adding a sizable two-body tunneling term 


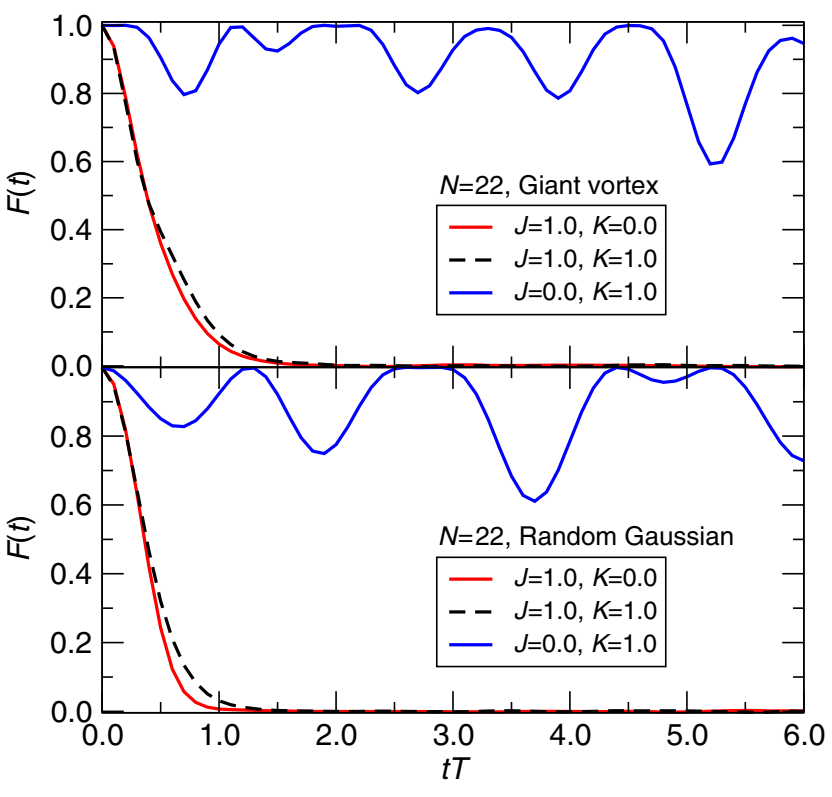

FIG. 8. Out-of-time-order correlators for our giant vortex system (top) and random Gaussian couplings (bottom). Nonzero temperature $T=1$ has been taken and other parameters as in Fig. 4. Correlators are displayed for a single disorder realization (unaveraged), but different realizations give very similar results for all interacting cases. Detailed shape of the oscillations apparent in the $J=0$ curves depends sensitively on the specific disorder realization, but all realizations show qualitatively similar behavior.

$K$ has only very modest effect on the behavior of $F(t)$ when the interaction strength is maintained. However, in the noninteracting case $(J=0, K=1)$, OTOC behavior changes qualitatively with the fast decay replaced by oscillations whose amplitude slowly increases.

\section{OUTLOOK: TOWARDS THE EXPERIMENTAL REALIZATION AND DETECTION OF THE SYK MODEL}

Our theoretical results we present above indicate that low-energy fermionic degrees of freedom in a device with geometry depicted in Fig. 1 provide a physical realization of the SYK model. Additionally, all the ingredients are currently in place to begin experimental explorations of the proposed system. Superconducting order has been induced and observed at the surface of several TI compounds by multiple groups [58-64]. Importantly, Ref. [62] already demonstrated the ability to tune the chemical potential in $\left(\mathrm{Bi}_{x} \mathrm{Sb}_{2-x}\right) \mathrm{Se}_{3}$ thin flakes through the neutrality point in the presence of superconductivity induced by Ti or Al contacts by a combination of chemical doping (tuning $x$ ) and backgate voltage. This is almost exactly what we require for the implementation of the SYK model. Well-developed techniques (such as focused ion milling) exist to fabricate patterns, such as a hole with an irregular shape, in a SC film deposited on the TI surface. In the remainder of this section we discuss in more detail the experimentally relevant constraints on the proposed device as well as possible ways to detect manifestations of the SYK physics in a realistic setting.

\section{A. Device geometry, length, and energy scales}

The key controllable design feature is the size of the hole, parametrized by its radius $R$. For simplicity, in the estimates below we assume a circular hole, but it should be understood that in a real experiment irregular shape is required to promote randomness of the zero-mode wave functions. For the desired number $N$ of Majorana zero modes, the hole must be large enough to pin $N$ vortices. Vortex pinning occurs because the SC order parameter $\Delta$ is suppressed to zero in the vortex core, which costs condensation energy. Vortices therefore prefer to occupy regions where $\Delta$ has been locally suppressed by defects, or in our case, by an artificially fabricated hole. The optimal hole size $R_{N}$ for $N$ vortices in our setup can thus be estimated from the requirement that all the electronic states inside the hole that reside within the SC gap are transformed into zero modes,

$$
\pi R_{N}^{2} \int_{-\Delta}^{\Delta} d \varepsilon D(\varepsilon)=N
$$

where $D(\varepsilon)=|\varepsilon| / 2 \pi v_{F}^{2} \hbar^{2}$ is the density of states of the TI surface. This gives

$$
R_{N}=\pi \xi \sqrt{2 N}
$$

with $\xi=\hbar v_{F} / \pi \Delta$ the BCS coherence length. In the absence of interactions, a hole of this size will produce an energy spectrum similar to that depicted in Fig. 4(b), with $N$ zero modes maximally separated from the rest of the spectrum.

In reality, if the SC film is in the type-II regime, a somewhat larger hole might be required to reliably pin $N$ vortices in a stable configuration and not create vortices nearby. The latter condition is that $B<B_{c 1}$, where $B_{c 1}$ is the lower critical field. Thus, the magnetic field to get the necessary flux is

$$
\pi\left(R_{N}+\lambda_{\text {eff }}\right)^{2} B=N \Phi_{0},
$$

where $\lambda_{\text {eff }}$ is the effective penetration depth of a thin SC film defined below Eq. (2.3). This gives

$$
R_{N} \geq \sqrt{\frac{N \Phi_{0}}{\pi B_{c 1}}}-\lambda_{\text {eff }}
$$

Taking the standard expression for the lower critical field, $B_{c 1}=\left(\Phi_{0} / 4 \pi \lambda_{\text {eff }}^{2}\right) K_{0}\left(\kappa_{\text {eff }}^{-1}\right)$, where $\kappa_{\text {eff }}=\lambda_{\text {eff }} / \xi$, Eq. (6.4) becomes 


$$
R_{N} \geq \lambda_{\text {eff }}\left(\sqrt{2 N / K_{0}\left(\kappa_{\mathrm{eff}}^{-1}\right)}-1\right),
$$

In the type-II regime, $\lambda_{\text {eff }}>\xi$ and Eq. (6.5) will generally imply larger hole size than Eq. (6.2). A larger hole size would reduce the spectral gap to some extent, but $N$ zero modes will remain robustly present. If the SC film remains in the type-I regime, then there is no additional constraint on $R_{N}$, but the applied field must be kept below the thermodynamic critical field $B_{c}$ of the film.

These considerations impose some practical constraints on the material composition and thickness $d$ of the SC film. In general, we want the film to be sufficiently thin so that scanning tunneling spectroscopy of the hole region can be performed. On the other hand, we want it to be either in the type-I or weakly type-II regime such that Eq. (6.5) does not enlarge the hole size significantly beyond the ideal radius given by Eq. (6.2). For $\mathrm{Pb}$, we have $\left(\xi, \lambda_{L}\right)=(83,37) \mathrm{nm}$. Taking $d=20 \mathrm{~nm}$ results in $\lambda_{\text {eff }} \simeq 137 \mathrm{~nm}$, and Eq. (6.5) imposes only a mild increase in the hole size compared to the ideal, which should not adversely affect the zero modes. For $\mathrm{Al}$, we have $\left(\xi, \lambda_{L}\right)=(1600,16) \mathrm{nm}$, and one can go down to very thin films and still remain in the type-I regime.

The TI film must be sufficiently thick so that it exhibits well-developed gapless surface states. For the $\mathrm{Bi}_{2} \mathrm{Se}_{3}$ family of materials this means thickness larger than 5 unit cells. TI films close to this critical thickness will also be easiest to bring to the neutrality point by backgating.

Using a hole close to the ideal size given by Eq. (6.2) will also promote the interaction strength. Intuitively, it is clear that screened Coulomb interaction between electrons will have maximum effect on the zero modes if their wave functions are packed as closely together as possible. With this in mind one can give a crude estimate of the expected interaction strength $J$ as follows. Starting from Eq. (2.20) with $V_{0}=2 \pi e^{2} \lambda_{\mathrm{TF}} / \epsilon$ and using Eq. (2.19) it is easy to show that

$$
J=\left(\frac{N^{3}}{3 !} \overline{J_{i j k l}^{2}}\right)^{1 / 2}=\sqrt{\frac{N^{3}}{6}} \frac{2 \pi e^{2} \lambda_{\mathrm{TF}}}{\epsilon \xi^{2}} \frac{12}{M_{s}^{3 / 2}},
$$

where we identify the length scale $\zeta$ with the SC coherence length $\xi$. We can obtain a physically more transparent expression by introducing the Bohr radius $a_{0}=\hbar^{2} / m_{e} e^{2} \simeq$ $0.52 \AA$ and the corresponding Rydberg energy $E_{0}=$ $e^{2} / 2 a_{0} \simeq 13.6 \mathrm{eV}$ :

$$
J=\frac{48 \pi}{\sqrt{6}} \sqrt{\frac{N^{3}}{M_{s}^{3}}}\left(\frac{a_{0} \lambda_{\mathrm{TF}}}{\epsilon \xi^{2}}\right) E_{0} .
$$

Several remarks are in order. Equation (6.7) implies that for a fixed hole size $R$ the coupling strength grows as $J \sim N^{3 / 2}$. It is therefore advantageous to put as many flux quanta in the hole as can be stabilized. For the "ideal" hole size $R=R_{N}$, given by Eq. (6.2), we have $M_{s}=2 \pi^{3} N$ and the dependence on $N$ drops out. The amplitude of $J$ will then depend only on the coherence length $\xi$, screening length $\lambda_{\mathrm{TF}}$, and dielectric constant $\epsilon$ of the system. To get an idea about the possible size of $J$ we assume $\lambda_{\mathrm{TF}} \approx \xi$ and $\epsilon \approx 50$, appropriate for the surface of a TI such as $\mathrm{Bi}_{2} \mathrm{Se}_{3}$. Equation (6.7) then gives $J \approx(1 \AA / \xi) 17.8 \mathrm{meV}$. It is clear that using a superconductor with a large gap and short coherence length would aid the observation of the SYK physics in this system at reasonable energy and temperature scales. Taking $\mathrm{Pb}$ as a concrete example, we have $\xi \simeq 52 \mathrm{~nm}$, for $d>20 \mathrm{~nm}$. Equation (6.5) does not impose additional restrictions on the hole diameter, and one obtains $J$ in the range of tens of $\mu \mathrm{eV}$. This energy scale is accessible to scanning tunneling spectroscopy (STS), which, as we argue below, constitutes the most convenient experimental probe.

\section{B. Experimental detection}

In our proposed setup the experimental detection of the signatures of the SYK state can be achieved using tunneling spectroscopy. Either a planar tunneling measurement with a fixed probe weakly coupled to the TI surface or a scanning tunnel probe can be used. STS has the advantage of simultaneously being able to image the topography of the device with nanoscale resolution and measure the tunneling conductance $g(\omega)$, which is proportional to the spectral function of the system $A(\omega)$. A recently developed technique [65] combines a STS tip with a miniature Hall probe, which allows additional measurement of the local magnetic field $B$ at the sample surface. Such a probe is ideally suited for the proposed SYK model setup as it can be used to independently determine the magnetic flux and thus the number $N$ of Majorana fermions in the system.

In the large- $N$ limit of the SYK model, $A(\omega)$ exhibits the characteristic $1 / \sqrt{|\omega|}$ singularity [illustrated in Fig. 2(a)], which should be easy to distinguish from the semicircle distribution that prevails in a system dominated by random two-fermion tunneling terms. In the large- $N$ limit and at sufficiently low temperature $k_{B} T \ll J$, the detection of the SYK behavior via tunneling spectroscopy should therefore be relatively straightforward.

In a realistic setup the number of flux quanta $N$ will be finite and perhaps not too large. In this case, our results in Fig. 7 show that the characteristic $1 / \sqrt{|\omega|}$ singularity is cut off such that $A(0)$ is finite and grows with $N$ only very slowly. Additional results assembled in Fig. 9 indicate that even in this situation it is possible to distinguish the interaction-dominated SYK behavior from the behavior characteristic of the weakly interacting system with random two-fermion couplings. For $J \gtrsim K$, we observe a relatively smooth spectral density peaked at $\omega=0$ characteristic of the strongly interacting regime. In the opposite limit $J \lesssim K$, 


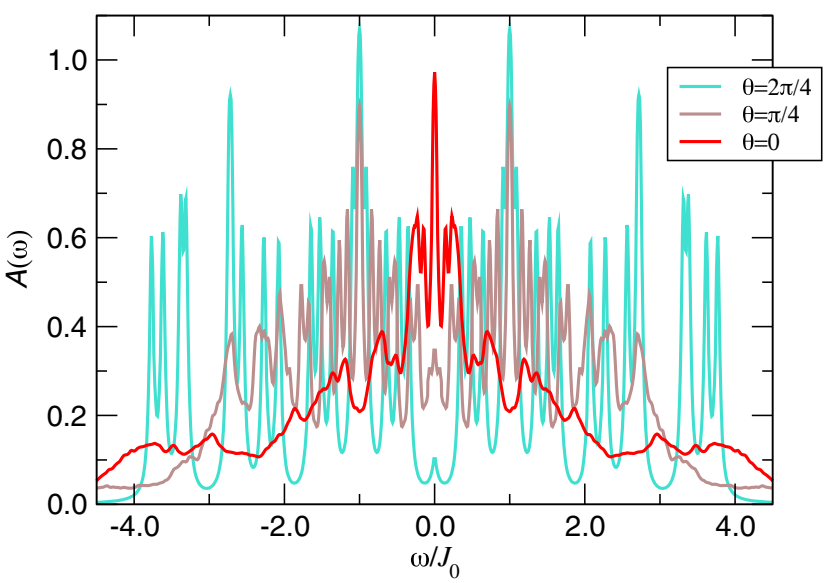

FIG. 9. Spectral function $A(\omega)$ calculated for the giant vortex geometry with $N=28$ and coupling constants $(J, K)=$ $J_{0}(\cos \theta, \sin \theta)$ taken to interpolate between the fully interacting and noninteracting limits.

nonuniversal fluctuations that strongly depend on the specific disorder realization become increasingly prominent. Eventually, when $J \ll K$, the spectral function consists of a series on $N$ sharp peaks. These peaks occur at the eigenvalues of the $N \times N$ random Hermitian matrix $i K_{i j}$ and represent the single-particle excitations of the noninteracting problem at $J=0$. At large $N$, these peaks merge to form a continuous distribution described by the semicircle law.

Full numerical diagonalization of the SYK Hamiltonian is feasible for $N$ up to 32 on a desktop computer and involves a matrix of size $2^{15} \times 2^{15}$ in each parity sector. By going to a supercomputer one can plausibly reach $N=42$ [66], but larger system sizes are out of reach due to the exponential growth of the Hamiltonian matrix with $N$. Experimental realization using the setup we propose here has no such limitation. Measurement of the spectral function in such a system could therefore help elucidate the approach to the large- $N$ limit in which the SYK model becomes analytically tractable by field theory techniques. This has relevance to the spontaneous breaking of the emergent conformal symmetry at large $N$ and a host of other interesting issues extensively discussed in the recent literature [6-13]. Measurement of the out-of-time-oder correlator $F(t)$ for $N$ larger than 32 could furthermore shed light on the emergence of the quantum chaotic behavior in the system, scrambling, and the dual relation to the extremal black hole in $\mathrm{AdS}_{2}$. A protocol to measure $F(t)$ in a system of this type is currently unknown, and this represents an interesting challenge and an opportunity for future study.

\section{CONCLUSIONS}

To conclude, we propose a physical realization of the Sachdev-Ye-Kitaev model that utilizes available materials and experimental techniques. The proposal is to use the surface of a 3D TI at its global neutrality point proximitized by a conventional superconductor with an irregular-shaped hole and magnetic flux threaded through the hole. We demonstrate that the conventional screened Coulomb interaction between electrons in such a setup leads to a Majorana fermion Hamiltonian at low energies with requisite random four-fermion couplings. Detailed analysis indicates behavior consistent with that expected of the SYK model. We give estimates for model parameters in the realistic systems and suggest experimental tests for the SYK behavior. This work thus provides connections between seemingly unrelated areas of researchmesoscopic physics, spin liquids, general relativity, and quantum chaos - and could lead to experimental insights into phemomena that are of great current interest.

\section{ACKNOWLEDGMENTS}

The authors are indebted to J. Alicea, O. Can, A. Kitaev, E. Lantagne-Hurtubise, M. Rozali, C.-M. Jian, I. Martin, and S. Sachdev for illuminating discussions. We thank Microsoft, NSERC, CIfAR, and Max Planck-UBC Centre for Quantum Materials for support. D. I. P. is grateful to KITP, where part of the research was conducted with support of the National Science Foundation Grant No. NSF PHY11-25915. Numerical simulations were performed in part with computer resources provided by WestGrid and Compute Canada Calcul.

[1] E.P. Wigner, On the Statistical Distribution of the Widths and Spacings of Nuclear Resonance Levels, Proc. Cambridge Philos. Soc. 47, 790 (1951).

[2] F. J. Dyson, Statistical Theory of the Energy Levels of Complex Systems. I, J. Math. Phys. (N.Y.) 3, 140 (1962).

[3] D. Sherrington and S. Kirkpatrick, Solvable Model of a Spin-Glass, Phys. Rev. Lett. 35, 1792 (1975).

[4] S. Sachdev and J. Ye, Gapless Spin-Fluid Ground State in a Random Quantum Heisenberg Magnet, Phys. Rev. Lett. 70, 3339 (1993).

[5] A. Camjayi and M. J. Rozenberg, Quantum and Thermal Fluctuations in the SU(n) Heisenberg Spin-Glass Model near the Quantum Critical Point, Phys. Rev. Lett. 90, 217202 (2003).

[6] A. Kitaev, in Proceedings of the KITP Program: Entanglement in Strongly-Correlated Quantum Matter, 2015, http:// online.kitp.ucsb.edu/online/entangled15/kitaev/, http:// online.kitp.ucsb.edu/online/entangled15/kitaev2/.

[7] J. Maldacena and D. Stanford, Remarks on the SachdevYe-Kitaev model, Phys. Rev. D 94, 106002 (2016).

[8] S. Sachdev, Bekenstein-Hawking Entropy and Strange Metals, Phys. Rev. X 5, 041025 (2015).

[9] J. Maldacena, S. H. Shenker, and D. Stanford, A Bound on Chaos, J. High Energy Phys. 08 (2016) 106.

[10] P. Hosur, X.-L. Qi, D. A. Roberts, and B. Yoshida, Chaos in Quantum Channels, J. High Energy Phys. 02 (2016) 004. 
[11] J. Polchinski and V. Rosenhaus, The Spectrum in the Sachdev-Ye-Kitaev Model, J. High Energy Phys. 04 (2016) 001.

[12] A. M. García-García and J. J. M. Verbaarschot, Spectral and Thermodynamic Properties of the Sachdev-Ye-Kitaev Model, Phys. Rev. D 94, 126010 (2016).

[13] Y.-Z. You, A. W. W. Ludwig, and C. Xu, Sachdev-Ye-Kitaev Model and Thermalization on the Boundary of Many-Body Localized Fermionic Symmetry Protected Topological States, Phys. Rev. B 95, 115150 (2017).

[14] W. Fu, D. Gaiotto, J. Maldacena, and S. Sachdev, Supersymmetric Sachdev-Ye-Kitaev Models, Phys. Rev. D 95, 026009 (2017).

[15] S. Banerjee and E. Altman, Solvable Model for a Dynamical Quantum Phase Transition from Fast to Slow Scrambling, Phys. Rev. B 95, 134302 (2017).

[16] Z. Bi, C.-M. Jian, Y.-Z. You, K. A. Pawlak, and C. Xu, Instability of the Non-Fermi Liquid State of the SachdevYe-Kitaev Model, Phys. Rev. B 95, 205105 (2017).

[17] Y. Gu, X.-L. Qi, and D. Stanford, Local Criticality, Diffusion and Chaos in Generalized Sachdev-Ye-Kitaev Models, J. High Energ. Phys. 05 (2017) 125.

[18] M. Berkooz, P. Narayan, M. Rozali, and J. Simón, Higher Dimensional Generalizations of the SYK Model, J. High Energy Phys. 01 (2017) 138.

[19] E. Witten, An SYK-Like Model Without Disorder, arXiv:1610.09758.

[20] I. Danshita, M. Hanada, and M. Tezuka, Creating and Probing the Sachdev-Ye-Kitaev Model with Ultracold Gases: Towards Experimental Studies of Quantum Gravity, arXiv:1606.02454.

[21] L. Garca-lvarez, I. L. Egusquiza, L. Lamata, A. del Campo, J. Sonner, and E. Solano, Digital Quantum Simulation of Minimal AdS/CFT, arXiv:1607.08560.

[22] J. Alicea, New Directions in the Pursuit of Majorana Fermions in Solid State Systems, Rep. Prog. Phys. 75, 076501 (2012).

[23] C. W. J. Beenakker, Search for Majorana Fermions in Superconductors, Annu. Rev. Condens. Matter Phys. 4, 113 (2013).

[24] M. Leijnse and K. Flensberg, Introduction to Topological Superconductivity and Majorana Fermions, Semicond. Sci. Technol. 27, 124003 (2012).

[25] T. D. Stanescu and S. Tewari, Majorana Fermions in Semiconductor Nanowires: Fundamentals, Modeling, and Experiment, J. Phys. Condens. Matter 25, 233201 (2013).

[26] S. R. Elliott and M. Franz, Colloquium: Majorana Fermions in Nuclear, Particle, and Solid-State Physics, Rev. Mod. Phys. 87, 137 (2015).

[27] V. Mourik, K. Zuo, S. M. Frolov, S. R. Plissard, E. P. A. M. Bakkers, and L. P. Kouwenhoven, Signatures of Majorana Fermions in Hybrid Superconductor-Semiconductor Nanowire Devices, Science 336, 1003 (2012).

[28] A. Das, Y. Ronen, Y. Most, Y. Oreg, M. Heiblum, and H. Shtrikman, Zero-Bias Peaks and Splitting in an Al-InAs Nanowire Topological Superconductor as a Signature of Majorana Fermions, Nat. Phys. 8, 887 (2012).

[29] M. T. Deng, C. L. Yu, G. Y. Huang, M. Larsson, P. Caroff, and H. Q. Xu, Anomalous Zero-Bias Conductance Peak in a
Nb-InSb Nanowire-Nb Hybrid Device, Nano Lett. 12, 6414 (2012).

[30] L. P. Rokhinson, X. Liu, and J. K. Furdyna, The Fractional ac Josephson Effect in a Semiconductor-Superconductor Nanowire as a Signature of Majorana Particles, Nat. Phys. 8, 795 (2012).

[31] A. D. K. Finck, D. J. Van Harlingen, P. K. Mohseni, K. Jung, and X. Li, Anomalous Modulation of a Zero-Bias Peak in a Hybrid Nanowire-Superconductor Device, Phys. Rev. Lett. 110, 126406 (2013).

[32] S. Hart, H. Ren, T. Wagner, P. Leubner, M. Mühlbauer, C. Brüne, H. Buhmann, L. W. Molenkamp, and A. Yacoby, Induced Superconductivity in the Quantum Spin Hall Edge, Nat. Phys. 10, 638 (2014).

[33] S. Nadj-Perge, I. K. Drozdov, J. Li, H. Chen, S. Jeon, J. Seo, A. H. MacDonald, B. Andrei Bernevig, and A. Yazdani, Observation of Majorana Fermions in Ferromagnetic Atomic Chains on a Superconductor, Science 346, 602 (2014).

[34] J.-P. Xu, M.-X. Wang, Z. L. Liu, J.-F. Ge, X. Yang, C. Liu, Z. A. Xu, D. Guan, C. L. Gao, D. Qian, Y. Liu, Q.-H. Wang, F.-C. Zhang, Q.-K. Xue, and Jin-Feng Jia, Experimental Detection of a Majorana Mode in the Core of a Magnetic Vortex inside a Topological Insulator-Superconductor $\mathrm{Bi}_{2} \mathrm{Te}_{3} / \mathrm{NbSe}_{2}$ Heterostructure, Phys. Rev. Lett. 114, 017001 (2015).

[35] H.-H. Sun, K.-W. Zhang, L.-H. Hu, C. Li, G.-Y. Wang, H.-Y. Ma, Z.-A. Xu, C.-L. Gao, D.-D. Guan, Y.-Y. Li, C. Liu, D. Qian, Y. Zhou, L. Fu, S.-C. Li, F.-C. Zhang, and J.-F. Jia, Majorana Zero Mode Detected with Spin Selective Andreev Reflection in the Vortex of a Topological Superconductor, Phys. Rev. Lett. 116, 257003 (2016).

[36] L. Fu and C. L. Kane, Superconducting Proximity Effect and Majorana Fermions at the Surface of a Topological Insulator, Phys. Rev. Lett. 100, 096407 (2008).

[37] J. C. Y. Teo and C. L. Kane, Topological Defects and Gapless Modes in Insulators and Superconductors, Phys. Rev. B 82, 115120 (2010).

[38] C.-K. Chiu, D. I. Pikulin, and M. Franz, Strongly Interacting Majorana Fermions, Phys. Rev. B 91, 165402 (2015).

[39] M. C. Gutzwiller, Chaos in Classical and Quantum Mechanics (Springer-Verlag, New York, 1990).

[40] F. Haake, Quantum Signatures of Chaos (Springer Science and Business Media, Secaucus, 2013), Vol. 54.

[41] A. Chew, A. Essin, and J. Alicea, Approximating the Sachdev-Ye-Kitaev Model with Majorana Wires, arXiv:1703.06890.

[42] A. Altland and M. R. Zirnbauer, Nonstandard Symmetry Classes in Mesoscopic Normal-Superconducting Hybrid Structures, Phys. Rev. B 55, 1142 (1997).

[43] R. Jackiw and P. Rossi, Zero Modes of the Vortex-Fermion System, Nucl. Phys. B190, 681 (1981).

[44] E. J. Weinberg, Index Calculations for the Fermion-Vortex System, Phys. Rev. D 24, 2669 (1981).

[45] L. Fu and C. L. Kane, Probing Neutral Majorana Fermion Edge Modes with Charge Transport, Phys. Rev. Lett. 102, 216403 (2009).

[46] A. R. Akhmerov, Johan Nilsson, and C. W. J. Beenakker, Electrically Detected Interferometry of Majorana Fermions 
in a Topological Insulator, Phys. Rev. Lett. 102, 216404 (2009).

[47] M. Cheng, R. M. Lutchyn, V. Galitski, and S. Das Sarma, Splitting of Majorana-Fermion Modes due to Intervortex Tunneling in a $p_{x}+i p_{y}$ Superconductor, Phys. Rev. Lett. 103, 107001 (2009).

[48] M. Cheng, R. M. Lutchyn, V. Galitski, and S. Das Sarma, Tunneling of Anyonic Majorana Excitations in Topological Superconductors, Phys. Rev. B 82, 094504 (2010).

[49] T. Liu and M. Franz, Electronic Structure of Topological Superconductors in the Presence of a Vortex Lattice, Phys. Rev. B 92, 134519 (2015).

[50] J. M. Murray and O. Vafek, Majorana Bands, Berry Curvature, and Thermal Hall Conductivity in the Vortex State of a Chiral p-Wave Superconductor, Phys. Rev. B 92 , 134520 (2015).

[51] D. I. Pikulin, C.-K. Chiu, X. Zhu, and M. Franz, InteractionEnabled Topological Phases in Topological InsulatorSuperconductor Heterostructures, Phys. Rev. B 92, 075438 (2015).

[52] H. B. Nielsen and M. Ninomiya, Absence of Neutrinos on a Lattice, Nucl. Phys. B185, 20 (1981).

[53] H. B. Nielsen and M. Ninomiya, A No-Go Theorem for Regularizing Chiral Fermions, Phys. Lett. B 105, 219 (1981).

[54] D. J. J. Marchand and M. Franz, Lattice Model for the Surface States of a Topological Insulator with Applications to Magnetic and Exciton Instabilities, Phys. Rev. B 86, 155146 (2012).

[55] M. Franz and Z. Tešanovć, Quasiparticles in the Vortex Lattice of Unconventional Superconductors: Bloch Waves or Landau Levels?, Phys. Rev. Lett. 84, 554 (2000).

[56] O. Vafek, A. Melikyan, M. Franz, and Z. Tešanović, Quasiparticles and Vortices in Unconventional Superconductors, Phys. Rev. B 63, 134509 (2001).

[57] W. Fu and S. Sachdev, Numerical Study of Fermion and Boson Models with Infinite-Range Random Interactions, Phys. Rev. B 94, 035135 (2016).

[58] G. Koren, T. Kirzhner, E. Lahoud, K. B. Chashka, and A. Kanigel, Proximity-Induced Superconductivity in
Topological $\mathrm{Bi}_{2} \mathrm{Te}_{2} \mathrm{Se}$ and $\mathrm{Bi}_{2} \mathrm{Se}_{3}$ Films: Robust ZeroEnergy Bound State Possibly due to Majorana Fermions, Phys. Rev. B 84, 224521 (2011).

[59] B. Sacépé, J. B. Oostinga, J. Li, A. Ubaldini, N. J. G. Couto, E. Giannini, and A. F. Morpurgo, Gate-Tuned Normal and Superconducting Transport at the Surface of a Topological Insulator, Nat. Commun. 2, 575 (2011).

[60] F. Qu, F. Yang, J. Shen, Y. Ding, J. Chen, Z. Ji, G. Liu, J. Fan, X. Jing, C. Yang, and L. Lu, Strong Superconducting Proximity Effect in $\mathrm{Pb}-\mathrm{Bi}_{2} \mathrm{Te}_{3}$ Hybrid Structures, Sci. Rep. 2, 339 (2012).

[61] J. R. Williams, A. J. Bestwick, P. Gallagher, Seung Sae Hong, Y. Cui, Andrew S. Bleich, J. G. Analytis, I. R. Fisher, and D. Goldhaber-Gordon, Unconventional Josephson Effect in Hybrid Superconductor-Topological Insulator Devices, Phys. Rev. Lett. 109, 056803 (2012).

[62] S. Cho, B. Dellabetta, A. Yang, J. Schneeloch, Z. Xu, T. Valla, G. Gu, M. J. Gilbert, and N. Mason, Symmetry Protected Josephson Supercurrents in Three-Dimensional Topological Insulators, Nat. Commun. 4, 1689 (2013).

[63] S.-Y. Xu, N. Alidoust, I. Belopolski, A. Richardella, C. Liu, M. Neupane, G. Bian, S.-H. Huang, R. Sankar, C. Fang, B. Dellabetta, W. Dai, Q. Li, M. J. Gilbert, F. Chou, N. Samarth, and M. Zahid Hasan, Momentum-Space Imaging of Cooper Pairing in a Half-Dirac-Gas Topological Superconductor, Nat. Phys. 10, 943 (2014).

[64] L. Zhao, H. Deng, I. Korzhovska, M. Begliarbekov, Z. Chen, E. Andrade, E. Rosenthal, A. Pasupathy, V. Oganesyan, and L. Krusin-Elbaum, Emergent Surface Superconductivity in the Topological Insulator $\mathrm{Sb}_{2} \mathrm{Te}_{3}$, Nat. Commun. 6, 8279 (2015).

[65] J.-Y.Ge, V. N. Gladilin, J. Tempere, C. Xue, J. T. Devreese, J. Van de Vondel, Y. Zhou, and V. V. Moshchalkov, Nanoscale Assembly of Superconducting Vortices with Scanning Tunnelling Microscope Tip, Nat. Commun. 7, 13880 (2016).

[66] S. Boixo, S. V. Isakov, V. N. Smelyanskiy, R. Babbush, N. Ding, Z. Jiang, J. M. Martinis, and H. Neven, Characterizing Quantum Supremacy in Near-Term Devices, arXiv:1608.00263. 